JOURNAL OF THE

AMERICAN MATHEMATICAL SOCIETY

Volume 13, Number 4, Pages 773-806

S 0894-0347(00)00342-8

Article electronically published on June 21, 2000

\title{
THE SPECTRA OF NONNEGATIVE INTEGER MATRICES VIA FORMAL POWER SERIES
}

KI HANG KIM, NICHOLAS S. ORMES, AND FRED W. ROUSH

\section{INTRODUCTION}

An old problem in matrix theory is to determine the $n$-tuples of complex numbers which can occur as the spectrum of a matrix with nonnegative entries (see BP94. Chapter 4] or [Min88, Chapter VII]). Authors have studied the case where the $n$ tuple is comprised of real numbers [Bor95, Cia68, Fri78, Kel71 Per53, Sal72, Sou83. Sul49, the case where the matrices under consideration are symmetric Fie74. JLL96], and the general problem [Joh81, LM99, LL79, Rea94, Rea96, Wuw97. Various necessary conditions and sufficient conditions have been provided, but a complete characterization is known for real $n$-tuples only for $n \leq 4$ [Kel71, Sul49] and for complex $n$-tuples only for $n \leq 3$ [LL79].

Motivated by symbolic dynamics, Boyle and Handelman refocused attention on the nonzero part of the spectrum by making the following "Spectral Conjecture" [BH91, BH93 (see also [Boy93, §8] and [LM95, Chapter 11]).

Below, a matrix $A$ is primitive if all entries of $A$ are nonnegative and for some $n$, all entries of $A^{n}$ are strictly positive. Also,

$$
\operatorname{tr}\left(\Lambda^{n}\right)=\sum_{i=1}^{d}\left(\lambda_{i}\right)^{n} \quad \text { and } \quad \operatorname{tr}_{n}(\Lambda)=\sum_{k \mid n} \mu\left(\frac{n}{k}\right) \operatorname{tr}\left(\Lambda^{k}\right)
$$

where $\mu$ is the Möbius function:

$$
\mu(n)= \begin{cases}1 & \text { if } n=1, \\ (-1)^{e} & \text { if } n \text { is the product of } e \text { distinct primes, } \\ 0 & \text { if } n \text { is divisible by } k^{2} \text { for some } k \geq 2\end{cases}
$$

Spectral Conjecture (Boyle, Handelman). Let $\Lambda=\left(\lambda_{1}, \lambda_{2}, \ldots, \lambda_{d}\right)$ be a d-tuple of nonzero complex numbers and let $S$ be a unital subring of $\mathbb{R}$. There is a primitive matrix over $S$ with characteristic polynomial $t^{m} \prod_{i=1}^{d}\left(t-\lambda_{i}\right)$ for some $m \geq 0$ if and only if

(1) the coefficients of the polynomial $\prod_{i=1}^{d}\left(t-\lambda_{i}\right)$ all belong to $S$,

(2) there exists $\lambda_{j}$ in $\Lambda$ such that $\lambda_{j}>\left|\lambda_{i}\right|$ for all $i \neq j$,

(3) if $S=\mathbb{Z}$, then $\operatorname{tr}_{n}(\Lambda) \geq 0$ for all $n \geq 1$,

$\left(3^{\prime}\right)$ if $S \neq \mathbb{Z}$, then for all $n \geq 1, \operatorname{tr}\left(\Lambda^{n}\right) \geq 0$ and for all $k \geq 1, \operatorname{tr}\left(\Lambda^{n}\right)>0$ implies $\operatorname{tr}\left(\Lambda^{n k}\right)>0$.

Received by the editors August 19, 1998 and, in revised form, February 1, 2000.

1991 Mathematics Subject Classification. Primary 15A18; Secondary 15A36, 58F03, 58F20.

Key words and phrases. Spectrum of nonnegative matrix, zeta function of subshift of finite type. 
It is not hard to show that these conditions are necessary. Moreover, the conditions can be easily verified for any example (condition (2) implies (3) or $\left(3^{\prime}\right)$ for all but finitely many $n, k)$.

Boyle and Handelman proved the conditions of the Spectral Conjecture to be sufficient in many cases, including the case $S=\mathbb{R}$ BH91, BH93. In this paper we resolve an important remaining case by proving the Spectral Conjecture for $S=\mathbb{Z}$ (Theorem 2.2). The Spectral Conjecture for $S=\mathbb{Q}$ (Corollary 2.3) follows from this result. Characterizations of the possible nonzero spectra of irreducible and general nonnegative matrices follow from the primitive case [BH91.

We begin by restating the argument for the necessity of the Boyle-Handelman conditions in the case $S=\mathbb{Z}$. Suppose $A$ is a primitive integer matrix with $\operatorname{det}(t \mathrm{I}-A)=t^{m} \prod_{i=1}^{d}\left(t-\lambda_{i}\right)$. Then condition (1) is clearly satisfied. Condition (2) follows from Perron-Frobenius Theory, the spectral radius of primitive matrix is always an eigenvalue of multiplicity one for that matrix. Condition (3) follows when we interpret $A$ as the adjacency matrix for a directed graph. In other words, if $A$ is a $k \times k$ matrix we can construct a directed graph $G_{A}$ with $k$ vertices such that the number of distinct edges from vertex $i$ to vertex $j$ is given by $A_{(i, j)}$. Here, the trace of $A^{n}$, given by $\operatorname{tr}\left(\Lambda^{n}\right)$, represents the number of loops of length $n$ in $G_{A}$, i.e., labelling each edge distinctly, $\operatorname{tr}\left(\Lambda^{n}\right)$ is the number of distinct edge sequences $e_{1} e_{2} \cdots e_{n}$ such that the terminal vertex of $e_{i}$ is equal to the initial vertex of $e_{i+1}$ and the terminal vertex of $e_{n}$ is the initial vertex of $e_{1}$. The $n$th net trace of $A$, given by $\operatorname{tr}_{n}(\Lambda)$, represents the number of loops of length $n$ in $G_{A}$ which are not formed by concatenating a single loop with itself.

Let $\Lambda=\left(\lambda_{1}, \lambda_{2}, \ldots, \lambda_{d}\right)$ satisfy the conditions of the Spectral Conjecture for $\mathbb{Z}$. We outline our construction of a matrix $A$ over $\mathbb{Z}_{+}$with nonzero spectrum $\Lambda$. First, we note that there is a primitive matrix $A(t)$ over $t \mathbb{Z}_{+}[t]$ (the ring of polynomials with nonnegative integer entries and no constant term) such that $\operatorname{det}(\mathrm{I}-A(t))=\prod_{i=1}^{d}\left(1-\lambda_{i} t\right)$ if and only if there is a primitive integer matrix $A$ with nonzero spectrum $\Lambda$ (see Boy93 $\$ 5]$ ). In Lemma [3.1] we use this fact to essentially reduce the problem of constructing a polynomial matrix $A(t)$ as above to finding polynomials $q_{1}, q_{2}, \ldots, q_{n} \in t \mathbb{Z}_{+}[t]$ and a power series $r \in t \mathbb{Z}_{+}[[t]]$ such that

$$
\prod_{i=1}^{d}\left(1-\lambda_{i} t\right)=(1-r(t)) \prod_{i=1}^{n}\left(1-q_{i}(t)\right) .
$$

In particular, we show that there are integers $o(n) \geq 0, n_{0} \geq 1$ and a polynomial $q \in t \mathbb{Z}_{+}[t]$ such that for each nonzero degree term in the power series

$$
\frac{\prod_{i=1}^{d}\left(1-\lambda_{i} t\right)}{(1-q(t)) \prod_{n=1}^{n_{0}}\left(1-t^{n}\right)^{o(n)}}
$$

the coefficient is nonpositive. We prove this by giving estimates of coefficients of the power series $\prod_{n=1}^{n_{0}}\left(1-t^{n}\right)^{-o(n)}$. We use different estimates for terms with degrees in linear, polynomial, subexponential, and exponential ranges of $n_{0}$. For sufficiently large $n_{0}$, these estimates imply the nonpositivity of coefficients of the above quotient up to a degree where the additional factor $1-q(t)$ takes over.

With the results here and in [BH91], a complete characterization of the spectra of primitive matrices over $\mathbb{R}$ or $\mathbb{Z}$ would follow from sharp bounds on the size of the realizing matrix. However, it seems that bounds of this type will be quite difficult 
to pin down. In particular, it follows from an inequality in [Joh81, LL79] that given any $N$ one can construct a 3-tuple satisfying the Boyle-Handelman conditions for $\mathbb{R}$ such that the size of a realizing matrix must be at least $N \times N$ (see [JLL96, LM98]). This is in dramatic contrast to the symmetric case where Johnson, Laffey and Loewy showed that if a $d$-tuple $\left(\lambda_{1}, \lambda_{2}, \ldots, \lambda_{d}\right)$ of real numbers is the nonzero spectrum of a symmetric matrix, then it is the nonzero spectrum of a symmetric matrix of size no more than $d(d+1) / 2$ [JLL96].

We propose that a variant of the size bound problem may be more approachable: Given $\left(\lambda_{1}, \lambda_{2}, \ldots, \lambda_{d}\right)$ satisfying the Boyle-Handelman conditions, produce sharp bounds on the size of a polynomial matrix $A(t)$ with $\operatorname{det}(\mathrm{I}-A(t))=\prod_{i=1}^{d}\left(1-\lambda_{i} t\right)$. In some respects, bounds of this type would be more useful than bounds on ordinary matrices since one can exhibit a much wider range of behavior with polynomial matrices of a fixed size. Moreover, it is conceivable that this line of study could lead to a solution to the original problem. A result of Perrin underscores the freedom afforded by polynomial matrices: For every $\lambda$ which occurs as the spectral radius of a primitive integer matrix, there is a $2 \times 2$ matrix $A(t)$ over $t \mathbb{Z}_{+}[t]$ with $\operatorname{det}(\mathrm{I}-A(t))=(1-\lambda t) \prod_{i=1}^{d}\left(1-\lambda_{i} t\right)$ and $\lambda>\left|\lambda_{i}\right|$ for all $i$ Per92 (see also Boy93. $\S 5])$.

Our characterization of the possible nonzero spectra of nonnegative matrices over $\mathbb{Z}$ translates into a characterization of the possible zeta functions for shifts of finite type in symbolic dynamics. In this setting, our work follows Lind's classification of entropies of shifts of finite type Lin84, Boyle and Handelman's classification of zeta functions for finitely presented systems [BH91] and various authors' development of polynomial matrices as tools in symbolic dynamics [BGMY80, KRW97, MT91. In general, there is a deep connection between symbolic dynamics and the asymptotic algebra of nonnegative matrices (see Boy93, BH93, LM95]). Boyle and Handelman's Generalized Spectral Conjecture (see [Boy93, §8]) concerns the matrix relation, strong shift equivalence over $R$, which in the case $R=\mathbb{Z}_{+}$corresponds to conjugacy between associated shifts of finite type. Strong shift equivalence over a semiring $R$ is defined as the transitive closure of the elementary relation $\sim_{R}$ where $A \sim_{R} B$ if there exist matrices $U, V$ over $R$ such that

$$
A=U V \quad \text { and } \quad V U=B .
$$

Strong shift equivalence seems to be the strongest asymptotic equivalence relation for matrices over a given semiring [Boy93, BH93]. Boyle and Handelman conjecture that a matrix $A$ over a unital ring $S \subseteq \mathbb{R}$ is strong shift equivalent over $S_{+}$to a primitive matrix if and only if the nonzero spectrum of $A$ satisfies the conditions of the Spectral Conjecture.

In Section 2 of this paper we provide statements of our results. In Section 3 we prove a reduction lemma and give a more detailed outline of the proof of the main theorem. The proof itself is contained in Sections 4-6. We thank Mike Boyle for many helpful discussions and for his detailed examination of portions of this manuscript. We also thank Doug Lind for his comments and for bringing the example from LM95, Chapter 11] to our attention (see Appendix B).

\section{Statement of the MAin RESUlts}

For a unital ring $S \subseteq \mathbb{R}$ we will often refer to the conditions of the Spectral Conjecture for $S$ as the Boyle-Handelman conditions for $S$. We will also clean 
up notation a bit by noting that for a matrix $A$, there is an $m \geq 0$ such that $\operatorname{det}(t \mathrm{I}-A)=t^{m} \prod_{i=1}^{d}\left(t-\lambda_{i}\right)$ if and only if $\operatorname{det}(\mathrm{I}-A t)=\prod_{i=1}^{d}\left(1-\lambda_{i} t\right)$.

Definition 2.1. A matrix $A$ over $\mathbb{R}_{+}$is primitive if there is an $n>0$ such that all of the entries of $A^{n}$ are positive.

Main Theorem 2.2. Let $\Lambda=\left(\lambda_{1}, \lambda_{2}, \ldots, \lambda_{d}\right)$ be a d-tuple of nonzero complex numbers with $\left|\lambda_{1}\right| \geq\left|\lambda_{2}\right| \geq \cdots \geq\left|\lambda_{d}\right|$. There exists a primitive integer matrix $A$ such that $\operatorname{det}(\mathrm{I}-A t)=\prod_{i=1}^{d}\left(1-\lambda_{i} t\right)$ if and only if

(1) the polynomial $\prod_{i=1}^{d}\left(1-\lambda_{i} t\right)$ has integer coefficients,

(2) $\lambda_{1}>\left|\lambda_{i}\right|$ for $i=2,3, \ldots, d$,

(3) $\operatorname{tr}_{n}(\Lambda) \geq 0$ for all $n \geq 1$.

As shown in [BH91], the Spectral Conjecture for $\mathbb{Q}$ follows from our main result.

Corollary 2.3. Let $\Lambda=\left(\lambda_{1}, \lambda_{2}, \ldots, \lambda_{d}\right)$ be a d-tuple of nonzero complex numbers with $\left|\lambda_{1}\right| \geq\left|\lambda_{2}\right| \geq \cdots \geq\left|\lambda_{d}\right|$. There is a primitive rational matrix $A$ with $\operatorname{det}(\mathrm{I}-A t)=\prod_{i=1}^{d}\left(1-\lambda_{i} t\right)$ if and only if

(1) the polynomial $\prod_{i=1}^{d}\left(1-\lambda_{i} t\right)$ has rational coefficients,

(2) $\lambda_{1}>\left|\lambda_{i}\right|$ for $i=2,3, \ldots, d$,

(3) for all $n \geq 1, \operatorname{tr}\left(\Lambda^{n}\right) \geq 0$ and

for all $k \geq 1, \operatorname{tr}\left(\Lambda^{n}\right)>0$ implies $\operatorname{tr}\left(\Lambda^{n k}\right)>0$.

We give a sketch of how the characterization of the spectra of irreducible and nonnegative matrices follows from the primitive case (see [BH91] for more details).

Definition 2.4. A matrix $A$ over $\mathbb{R}_{+}$is irreducible if for all $(i, j)$ there is an $n \geq 0$ such that the $(i, j)$ entry of $A^{n}$ is positive.

If a matrix $A$ is irreducible, then there is a $p \geq 1$ and a primitive matrix $B$ such that $\operatorname{det}(\mathrm{I}-A t)=\operatorname{det}\left(\mathrm{I}-B t^{p}\right)$. This leads to the following.

Corollary 2.5. Let $\Lambda=\left(\lambda_{1}, \lambda_{2}, \ldots, \lambda_{d}\right)$ be a d-tuple of nonzero complex numbers. There exists an irreducible matrix $A$ over $\mathbb{Z}(\mathbb{Q})$ such that $\operatorname{det}(\mathrm{I}-A t)=\prod_{i=1}^{d}\left(1-\lambda_{i} t\right)$ if and only if there exist an integer $p>0$ and a partition of $\Lambda$ into subtuples $\left\{\Lambda_{k}: 0 \leq k<p\right\}$ such that

(1) $\Lambda_{k+1}=e^{2 \pi i / p} \Lambda_{k}$ for $0 \leq k<(p-1)$,

(2) $\left(\Lambda_{0}\right)^{p}$ satisfies the Boyle-Handelman conditions for $\mathbb{Z}(\mathbb{Q})$.

If $A$ is a nonnegative matrix, then there are irreducible matrices $A_{1}, A_{2}, \ldots, A_{m}$ such that $\operatorname{det}(\mathrm{I}-A t)=\prod_{j=1}^{m} \operatorname{det}\left(\mathrm{I}-A_{j} t\right)$.

Corollary 2.6. Let $\Lambda=\left(\lambda_{1}, \lambda_{2}, \ldots, \lambda_{d}\right)$ be a d-tuple of nonzero complex numbers. There exists a nonnegative matrix $A$ over $\mathbb{Z}(\mathbb{Q})$ such that $\operatorname{det}(\mathrm{I}-A t)=$ $\prod_{i=1}^{d}\left(1-\lambda_{i} t\right)$ if and only if there exist an integer $n>0$, integers $p(1), p(2), \ldots, p(n)$ $>0$ and a partition of $\Lambda$ into subtuples $\left\{\Lambda_{(j, k)}: 1 \leq j \leq n, 0 \leq k<p(j)\right\}$ such that

(1) $\Lambda_{(j, k+1)}=e^{2 \pi i / p(j)} \Lambda_{(j, k)}$ for all $0 \leq k<(p(j)-1)$ and $1 \leq j \leq n$,

(2) $\left(\Lambda_{(j, 0)}\right)^{p(j)}$ satisfies the Boyle-Handelman conditions for $\mathbb{Z}(\mathbb{Q})$ for all $1 \leq j \leq$ $n$.

Remark 2.7. A characterization of zeta functions for shifts of finite type follows from Corollary 2.6. Suppose $A$ is a nonnegative matrix $A$ and $\sigma_{A}$ is the corresponding shift of finite type. If $\operatorname{Fix}\left(\left(\sigma_{A}\right)^{n}\right)$ is the set of fixed points of the map 
$\left(\sigma_{A}\right)^{n}$, then we have the following definition and result:

$$
\zeta_{\left(\sigma_{A}\right)}(t):=\exp \left(\sum_{n=1}^{\infty} \frac{\# \operatorname{Fix}\left(\left(\sigma_{A}\right)^{n}\right)}{n} t^{n}\right)=\frac{1}{\operatorname{det}(\mathrm{I}-A t)} .
$$

Therefore, a power series in $\mathbb{Z}[[t]]$ is a zeta function if and only if it is equal to $\prod_{i=1}^{d}\left(1-\lambda_{i} t\right)^{-1}$ where $\left(\lambda_{1}, \lambda_{2}, \ldots, \lambda_{d}\right)$ satisfies the conditions of Corollary 2.6 Moreover, since irreducible matrices give rise to irreducible shifts of finite type and primitive matrices give rise to mixing shifts of finite type, Theorem 2.2 and Corollary 2.5 give finer characterizations for zeta functions associated to shifts of finite type in those classes.

\section{PROOF SCHEME FOR THE MAIN THEOREM}

For the remainder of the paper, suppose $\Lambda=\left(\lambda_{1}, \lambda_{2}, \ldots, \lambda_{d}\right)$ satisfies the BoyleHandelman conditions for $\mathbb{Z}$ and $1-p(t)=\prod_{i=1}^{d}\left(1-\lambda_{i} t\right)$. Let $\lambda=\lambda_{1}$.

We begin this section by showing that the problem of creating a primitive matrix $A$ over $\mathbb{Z}$ with $\operatorname{det}(\mathrm{I}-A t)=1-p(t)$ reduces to writing

$$
1-p(t)=(1-r(t)) \prod_{i=1}^{n}\left(1-q_{i}(t)\right)
$$

where $q(t) \in t \mathbb{Z}_{+}[t]$ and $r(t) \in t \mathbb{Z}_{+}[[t]]$. The reduction uses in a critical way the fact that there is a primitive matrix $A$ with entries in $\mathbb{Z}_{+}$such that $\operatorname{det}(\mathrm{I}-A t)=1-p(t)$ if and only if there is a primitive polynomial matrix $A(t)$ with entries in $t \mathbb{Z}_{+}[t]$ such that $\operatorname{det}(\mathrm{I}-A(t))=1-p(t)$ (see Boy93 for details).

3.1. Main reduction. Before we justify the reduction to the factorization problem in formal power series, we prove a lemma about coefficients of large degree in a rational power series.

Lemma 3.1. Suppose $f(t) \in \mathbb{Z}[t], u(t) \in \mathbb{R}[t]$ and $\alpha>0$ such that all roots of $u(t)$ have modulus greater than $1 / \alpha$. Let $r(t)=\sum r_{n} t^{n}$ be the power series

$$
r(t)=\frac{f(t)}{(1-\alpha t) u(t)} .
$$

Then there exist constants $K, k>0$ such that

$$
\left|\frac{r_{n}}{\alpha^{n}}-\frac{f(1 / \alpha)}{u(1 / \alpha)}\right|<K e^{-k n} \quad \text { for } n \geq 0 .
$$

Proof. Let $a=f(1 / \alpha) / u(1 / \alpha)$. Then $1 / \alpha$ is a root of $f(t)-a u(t)$ and $b(t)=$ $(f(t)-a u(t)) /(1-\alpha t)$ is a polynomial. We may rewrite $r(t)$ as

$$
r(t)=\frac{a}{1-\alpha t}+\frac{b(t)}{u(t)}=a \sum_{n=0}^{\infty} \alpha^{n} t^{n}+\frac{b(t)}{u(t)} .
$$

Let $b(t) / u(t)=\sum c_{n} t^{n}$. By the condition on the roots of $u(t)$, the radius of convergence of the power series $\sum c_{n} t^{n}$ is larger than $1 / \alpha$. Therefore there exist constants $K, k>0$ such that $\left|c_{n}\right| \alpha^{-n}<K e^{-k n}$. Since $r_{n}=a \alpha^{n}+c_{n}$, the result follows. 
If, as in the previous lemma, a polynomial has a factorization of the form $(1-\alpha t) u(t)$ where the modulus of all roots of $u(t)$ are larger than $1 / \alpha$, we will call $\alpha$ the Perron value of the polynomial $(1-\alpha t) u(t)$. If $q \in t \mathbb{Z}_{+}[t]$ and the degrees of terms in $q(t)$ with nonzero coefficients have greatest common divisor one, then $1-q(t)$ has such a factorization.

We now show that producing a polynomial matrix $A(t)$ over $t \mathbb{Z}_{+}[t]$ with $\operatorname{det}(\mathrm{I}-A(t))=1-p(t)$ reduces to producing a certain factorization of $1-p(t)$ in $\mathbb{Z}[[t]]$.

Main Reduction Lemma. Let $p \in t \mathbb{Z}[t]$. If there exist polynomials $q_{1}, q_{2}, \ldots, q_{m}$ in $t \mathbb{Z}_{+}[t]$ and a power series $r$ in $t \mathbb{Z}_{+}[[t]]$ such that

(1) there is a Perron value $\alpha$ for the polynomial $\prod_{j=1}^{m}\left(1-q_{j}(t)\right)$,

(2) $1-p(1 / \alpha)<0$,

(3) $1-p(t)=(1-r(t)) \prod_{j=1}^{m}\left(1-q_{j}(t)\right)$ in $\mathbb{Z}[[t]]$,

then there exists a matrix $A(t)$ with entries in $t \mathbb{Z}_{+}[t]$ such that

$$
\operatorname{det}(\mathrm{I}-A(t))=1-p(t)
$$

Proof. Assume that the polynomials $q_{i}$ are numbered so that $1 / \alpha$ is a root of the first polynomial $1-q_{1}(t)$. Then $1-q_{1}(t)$ factors as $(1-\alpha t) u(t)$ where $u(1 / \alpha)=$ $q_{1}^{\prime}(1 / \alpha) / \alpha>0$. Note that for $j \geq 2$ we have $1-q_{j}(1 / \alpha)>0$. This follows from $1-q_{j}(0)=1$ and assumption (1) which implies that the positive number $1 / \alpha$ is strictly smaller than the unique positive root of $1-q_{j}(t)$.

Since $1-p(1 / \alpha)<0$ and $1-q_{j}(1 / \alpha)>0$ for all $j \geq 2$, it follows from Lemma 3.1 that there is an $N>0$ such that for all $1 \leq k \leq m$, the $n$th coefficient of $(1-p(t)) \prod_{j=1}^{k}\left(1-q_{j}(t)\right)^{-1}$ is negative for all $n>N$. Select this $N$ to exceed the degree of $p(t)$, the degree of all polynomials $q_{j}(t)$ and $m$.

Let $a_{0}(t)=p(t)$ and for $1 \leq k \leq m$ let $1-a_{k}(t)$ be the sum of terms in the power series $(1-p(t)) \prod_{j=1}^{k}\left(1-q_{j}(t)\right)^{-1}$ up to degree $2 N$. Let $b_{k}(t)$ be the polynomial defined by the relation

$$
1-a_{k-1}(t)=\left(1-a_{k}(t)\right)\left(1-q_{k}(t)\right)-b_{k}(t) .
$$

Since the first $2 N$ terms of $1-a_{k-1}(t)$ and $\left(1-a_{k}(t)\right)\left(1-q_{k}(t)\right)$ agree, all coefficients of $b_{k}(t)$ in degrees $2 N$ and less are zero. In degrees greater than $2 N$, the coefficients of $b_{k}(t)$ equal the coefficients of the product $a_{k}(t) q_{k}(t)$. By the choice of $N, q_{k}(t) \in \mathbb{Z}_{+}[t]$ has degree at most $N$ and $a_{k}(t)$ has nonnegative coefficients in degrees $(N+1)$ and higher. Therefore, all coefficients of $b_{k}(t)$ are nonnegative.

Beginning with $1-p(t)=1-a_{0}(t)$ and successively using equation (3.1), we have

$$
1-p(t)=\left(1-a_{m}(t)\right) \prod_{j=1}^{m}\left(1-q_{j}(t)\right)-\sum_{i=1}^{m}\left[b_{i}(t) \prod_{j=1}^{i-1}\left(1-q_{j}(t)\right)\right] .
$$

It follows from the hypotheses of the lemma that the polynomial $a_{m}(t)$ has all nonnegative coefficients since it is equal to the first $2 N$ terms of the power series $r(t)$. 
Now let $M(t)$ be the following polynomial matrix over $\mathbb{Z}_{+}[t]$ :

$$
M(t)=\left(\begin{array}{ccccc}
a_{m}(t) & 1 & 0 & \cdots & 0 \\
b_{m}(t) & q_{m}(t) & 1 & \ddots & \vdots \\
b_{m-1}(t) & 0 & q_{m-1}(t) & \ddots & 0 \\
\vdots & \vdots & \ddots & \ddots & 1 \\
b_{1}(t) & 0 & \ldots & 0 & q_{1}(t)
\end{array}\right) .
$$

Using equation (3.2), we find $\operatorname{det}(\mathrm{I}-M(t))=1-p(t)$.

Finally, we replace each occurrence of 1 in $M(t)$ with a $t$ and each $b_{i}(t)$ with $b_{i}(t) / t^{m+1-i}$. The result is a matrix $A(t)$ over $t \mathbb{Z}_{+}[t]$ with $\operatorname{det}(\mathrm{I}-A(t))=1-$ $p(t)$.

We now turn our attention to producing a factorization of our candidate polynomial $1-p(t)$ as in the Main Reduction Lemma. One can recursively define a sequence of integers $o(i)$ such that $1-p(t)$ has a formal factorization as

$$
1-p(t)=\prod_{i=1}^{\infty}\left(1-t^{i}\right)^{o(i)}
$$

We claim $o(i)=\operatorname{tr}_{i}(\Lambda) / i \geq 0$ for all $i$. To see this, first note the relation

$$
1-p(t)=\exp \left(\sum_{j=1}^{d} \log \left(1-\lambda_{j} t\right)\right)=\exp \left(-\sum_{i=1}^{\infty} \frac{\operatorname{tr}\left(\Lambda^{i}\right)}{i} t^{i}\right) .
$$

Taking logarithms and comparing coefficients in the resulting power series, we see $\operatorname{tr}\left(\Lambda^{i}\right)=\sum_{k \mid i} k o(k)$. Since $\operatorname{tr}\left(\Lambda^{i}\right)=\sum_{k \mid i} \operatorname{tr}_{k}(\Lambda)$, we obtain $o(i)=\operatorname{tr}_{i}(\Lambda) / i$.

Thus we use the Net Trace Condition to obtain an infinite factorization as above with nonnegative exponents $o(i)$. Moreover, we will know from the Perron condition that, for large $i, i o(i) \lambda^{-i} \approx 1$.

It seems natural to attempt to truncate the infinite factorization and write

$$
1-p(t)=(1-r(t)) \prod_{i=1}^{n_{0}}\left(1-t^{i}\right)^{o(i)}
$$

where $r \in t^{n_{0}+1} \mathbb{Z}_{+}[[t]]$. With such a factorization, we automatically have that the first $n_{0}$ coefficients of $r(t)$ are zero, and coefficients in degrees $n_{0}$ through $2 n_{0}$ are positive. In fact we can use this truncated product approach to factor many examples, including $1-4 t+6 t^{2}-6 t^{3}$ from LM95 (see Appendix B). This example was presented as one which satisfies the Boyle/Handelman conditions, but one for which there was no known matrix with the corresponding nonzero spectrum.

However, we are not able to factor general polynomials as above. In particular, to apply the Main Reduction Lemma, we need the denominator polynomial to have a Perron value in an appropriate region to control coefficients of large degree for the quotient power series.

The final factorization we seek will be one of the form

$$
1-p(t)=(1-r(t))(1-q(t)) \prod_{i=1}^{n_{0}}\left(1-t^{i}\right)^{o(i)}
$$

where $q(t) \in t \mathbb{Z}_{+}[t]$ and $r(t) \in t^{n_{0}+1} \mathbb{Z}_{+}[[t]]$. We are assuming $1-p(t)$ has a Perron value $\lambda$. If we select $1-q(t)$ so that its Perron value $\alpha$ is smaller than $\lambda$ but larger 
than $\left|\lambda_{i}\right|$ for $i>1$, then we can guarantee $1-p(1 / \alpha)<0$. Thus by Lemma 3.1 all coefficients of large degree terms $r(t)$ have the correct sign.

We know the $n$th coefficient of $(1-p(t)) /(1-q(t)) \prod_{i=1}^{n_{0}}\left(1-t^{i}\right)^{o(i)}$ has the appropriate sign when $n$ is small (less than $2 n_{0}$ ) and when $n$ is sufficiently large. The difficulty lies in bridging the gap between these two regions. The proof we present relies on careful estimates of the coefficients of rational power series in various ranges. For example, we use different arguments for $n \in\left(2 n_{0}+\log ^{2} n_{0}, n_{0}^{20}\right)$, $n \in\left[n_{0}^{20}, e^{r n_{0}}\right)$ and $n \in\left[e^{r n_{0}}, \infty\right)$. The sequence of lemmas is somewhat long, and we present now an overview which we hope will help the reader digest the proof.

3.2. Overview of the estimates. We wish to show that the $n$th coefficient of the product of the polynomial $1-p(t)$ with the power series $(1-q(t))^{-1} \prod_{i=1}^{n_{0}}\left(1-t^{i}\right)^{-o(i)}$ is nonpositive for $n>0$. To do so we will follow one of two arguments.

Let $c(t)=\sum_{i=0}^{\infty} c_{n} t^{n}$ be any power series with $c_{0}=1$ and $c_{n} \geq 0$ for all $n$. Suppose we would like to show that the $n$th coefficient of $(1-p(t)) c(t)$ is negative. We may write the $n$th coefficient of the product of $(1-p(t)) c(t)$ as $c_{n}\left(1-\sum_{i=1}^{d} p_{i} c_{n-i} / c_{n}\right)$.

To apply the Difference Argument, we show that there is a $D>0$ such that $c_{n-i} / c_{n} \approx \lambda^{-i}(1+i D)$ for $i=1,2, \ldots, d$. Then

$$
1-\sum_{i=1}^{d} p_{i} c_{n-i} / c_{n} \approx \underbrace{1-\sum_{i=1}^{d} p_{i} \lambda^{-i}}_{0}-D \lambda^{-1} \underbrace{\sum_{i=1}^{d} i p_{i} \lambda^{-i+1}}_{p^{\prime}(1 / \lambda)} .
$$

Since $p^{\prime}(1 / \lambda)>0$, the expression on the right-hand side is negative. In order to make this argument precise, we will have to show that the error in the approximation is small compared to the number $D$.

To apply the Ratio Argument, we show that there is a number $\alpha<\lambda$ such that $c_{n-i} / c_{n} \approx \alpha^{-i}$ for $i=1,2, \ldots, d$ and $1-p(1 / \alpha)<0$. Then

$$
1-\sum_{i=1}^{d} p_{i} c_{n-i} / c_{n} \approx 1-\sum_{i=1}^{d} p_{i} \alpha^{-i}=1-p(1 / \alpha) .
$$

To make this argument precise, we will have to show that the error in the approximation is small compared to $1-p(1 / \alpha)$. By the Mean Value Theorem, this will reduce to showing that the error in the approximation is small compared to $|\lambda-\alpha|$.

In Section 4, we examine the product of the power series

$$
S(t)=\sum_{n=0}^{\infty} S_{n} t^{n}=\prod_{i=1}^{n_{0}}\left(1-t^{i}\right)^{-o(i)}
$$

and the polynomial $1-p(t)$. We apply the Difference Argument to show that the $n$th coefficient of this product is negative for $n \in\left(n_{0}, n_{0}^{20}\right)$. To do so, we first give bounds on $S_{n} / S_{n+1}$ for $n$ in an initial range. Then we introduce a different power series $E(t)$, the coefficients of which approximate the coefficients of $S(t)$. Let

$$
E(t)=\sum_{n=0}^{\infty} E_{n} t^{n}=\exp \left(\sum_{i=1}^{n_{0}} \frac{\operatorname{tr}\left(\Lambda^{i}\right)}{i} t^{i}\right) .
$$


By differentiating $E(t)$, we find that the coefficients of $E(t)$ satisfy a recursion relation:

$$
n E_{n}=\sum_{i=1}^{n_{0}} \operatorname{tr}\left(\Lambda^{i}\right) E_{n-i}
$$

With the estimates on ratios of $S_{n} / S_{n+1}$ as the base case, we use the recursion formula to give estimates on $E_{n} / E_{n+1}$ for $n$ up to some exponential function of $n_{0}$. We show that for large $n_{0}$, all approximations introduce errors which are small compared to $D=\lambda S_{n-1} / S_{n}-1>0$ for $n \in\left(n_{0}, n_{0}^{20}\right)$. Then by the Difference Argument the series $(1-p(t)) S(t)$ has nonnegative coefficients in this polynomial range.

In Section 5 we make further use of the recursion formula for $E_{n}$ and apply the Ratio Argument to the $n$th coefficient of $S(t)(1-p(t))$ for $n \in\left[n_{0}^{20}, e^{r n_{0}}\right)(r$ is a number chosen based on other constants). Here we think of the recursion relation on the coefficients of $E(t)$ as being given by a matrix. In other words, there is a matrix $A_{n}$ such that

$$
\left(E_{n}, E_{n-1}, \ldots, E_{n-n_{0}+1}\right)^{\mathrm{T}}=A_{n}\left(E_{n-1}, E_{n-2}, \ldots, E_{n-n_{0}}\right)^{\mathrm{T}} .
$$

Thus, beginning with an initial string of $n_{0}$ consecutive coefficients, we obtain a string of coefficients of larger degree by applying the matrix $A_{n} A_{n-1} \cdots A_{n-N+1}$. As we will show for $n>n_{0}^{4}$ and $N=n_{0}^{6}$, applying this product of matrices to a vector $\mathbf{v} \in \mathbb{R}_{+}^{n_{0}}$ is approximately the same as multiplying by the $N$ th power of the single matrix $A_{n}$ to $\mathbf{v}$. The matrix $A_{n}$ has a Perron eigenvalue $\alpha_{n}$. A fairly general argument involving estimates of moduli of other eigenvalues of $A_{n}$ implies that when a large power of $A_{n}$ is applied to $\mathbf{v}$ the result is a vector which is very close to an eigenvector for $A_{n}$ corresponding to $\alpha_{n}$. Since eigenvectors corresponding to $\alpha_{n}$ are exactly the vectors with ratios of successive entries equal to $\alpha_{n}$, we see that $E_{n} / E_{n-1} \approx 1 / \alpha_{n}$. Applying the Ratio Argument, all of these approximations taken with a lower bound on $\lambda-\alpha_{n}$ imply that the $n$th coefficient of $S(t)(1-p(t))$ is negative for $n \in\left[n_{0}^{20}, e^{r n_{0}}\right)$.

In Section [6] we incorporate an additional factor $(1-q(t))^{-1}$ into the factorization scheme. We first select a real number $\beta$ such that $\beta<\lambda$ and $1-p(1 / \beta)<0$. Then let $1-q(t)$ be an integer polynomial approximation of

$$
1-\frac{1}{n_{0}^{3}-n_{0}^{2}}\left(\beta^{n_{0}^{2}+1} t^{n_{0}^{2}+1}+\beta^{n_{0}^{2}+2} t^{n_{0}^{2}+2}+\cdots+\beta^{n_{0}^{3}} t^{n_{0}^{3}}\right) .
$$

The Perron value for $1-q(t)$ is approximately $\beta$. Using the matrix arguments from Section 5 on the recursion formula for coefficients of $(1-q(t))^{-1}$, we show that ratios of successive coefficients of $(1-q(t))^{-1}$ are approximately $\beta$ for $n>n_{0}^{20}$. Again we can apply the Ratios Argument to show that the $n$th coefficient of $(1-p(t)) /(1-q(t))$ is negative for $n>n_{0}^{20}$.

Finally, we combine all of the estimates to show that the $n$th coefficient of the product $(1-p(t)) S(t)(1-q(t))^{-1}$ is nonpositive for all $n>0$. We think of the $n$th coefficient of $(1-p(t)) S(t)(1-q(t))^{-1}$ either as the $n$th coefficient of the product of $(1-p(t)) S(t)$ and $(1-q(t))^{-1}$ or as the $n$th coefficient of the product of $S(t)$ and $(1-p(t))(1-q(t))^{-1}$. In either case, we will write the $n$th coefficient as the sum of a large number of negative terms and a relatively small number of positive terms. We are able to conclude that the overall sum is negative. 


\section{Polynomial Range}

In this section we follow the Difference Argument to show that if $n_{0}$ is sufficiently large and $n \in\left(n_{0}, n_{0}^{20}\right)$, then the $n$th coefficient of the power series $S(t)(1-p(t))$ is bounded above by $S_{n}$ times a negative constant over $n_{0}$ (Polynomial Range Lemma). We must show that for each $n \in\left(n_{0}, n_{0}^{20}\right)$ there is a number $D>0$ such that $S_{n-i} / S_{n} \approx \lambda^{-i}(1+i D)$ for $i=1,2, \ldots, d$. We will let $D=\lambda S_{n-1} / S_{n}-1$.

It will be convenient for us to write many of our estimates in terms of coefficients scaled by $\lambda$. Our convention will be to write coefficients of power series with capital letters and scaled coefficients with lower case letters. For example, let $s_{n}=S_{n} \lambda^{-n}$. Let $Z(t)$ denote the candidate zeta function $Z(t)=\sum_{i=1}^{\infty} z_{n}(\lambda t)^{n}=(1-p(t))^{-1}$.

Throughout the remainder of the paper, we will use the fact that there exist constants $a, K_{0}, k_{0}>0$ such that

$$
\left|z_{n}-a\right|<K_{0} e^{-k_{0} n} \text { and }\left|n o(n) \lambda^{-n}-1\right|<K_{0} e^{-k_{0} n}
$$

for all $n$.

In the following lemmas we use the term "constant" or "universal constant" to mean a number which is chosen based solely on $1-p(t)$. In particular, it is important that constants do not depend upon the choice of $n_{0}$. Many constants used in the statements of lemmas will be denoted by $K$ or $k$ and will be numbered so as to distinguish them.

4.1. Initial bounds. In the following two lemmas, our goal is to show that $s_{n} / s_{n+1}$ -1 is bounded between two positive constants times $1 / n_{0}$ for $n \in\left[n_{0}+\log ^{2} n_{0}, 2 n_{0}+\right.$ $\left.\log ^{2} n_{0}\right]$. In Section 4.2 we will use these initial bounds along with a recursive formula to produce estimates on $s_{n} / s_{n+1}-1$ for larger $n$.

We begin by proving a uniform lower bound on the coefficients $s_{n}$ for $n$ in this initial range.

Lemma 4.1. There exists a constant $K_{1}>0$ such that if $n_{0}$ is sufficiently large, then

$$
s_{n}>K_{1} \quad \text { for } \quad n \in\left(n_{0}, 2 n_{0}+\log ^{2} n_{0}\right]
$$

Proof. We have the following expressions for $S(t)$ :

$$
S(t)=\prod_{i=1}^{n_{0}}\left(1-t^{i}\right)^{-o(i)}=\prod_{i=n_{0}+1}^{\infty}\left(1-t^{i}\right)^{o(i)} /(1-p(t)) .
$$

Thus for $n \in\left(n_{0}, 2 n_{0}+\log ^{2} n_{0}\right]$, we have

$$
s_{n}=z_{n}-\sum_{i=n_{0}+1}^{n} \frac{o(i)}{\lambda^{i}} z_{n-i}+\sum_{\substack{i, j>n_{0} \\
i+j \leq n}} \frac{o(i) o(j)}{\lambda^{i+j}} z_{n-i-j}+\sum_{i=n_{0}+1}^{n / 2} \lambda^{-2 i}\left(\begin{array}{c}
o(i) \\
2
\end{array}\right) z_{n-2 i} .
$$

(The last two summations only occur when $n \geq 2 n_{0}+2$.) Since the last two terms summations above are nonnegative,

$$
s_{n} \geq z_{n}-\sum_{i=n_{0}+1}^{n} \frac{o(i)}{\lambda^{i}} z_{n-i}=z_{n}\left(1-\sum_{i=n_{0}+1}^{n} \frac{o(i) z_{n-i}}{\lambda^{i} z_{n}}\right) .
$$

From estimates on $z_{n}$ and $o(n)$, the difference between the summation of the terms $\sum_{i=n_{0}+1}^{n} o(i) z_{n-i} / \lambda^{i} z_{n}$ and $\sum_{i=n_{0}+1}^{n} 1 / i$ is at most a constant times $1 / n_{0}$. 
Choose $n_{0}$ large enough so that

$$
\sum_{i=n_{0}+1}^{n} \frac{1}{i}<\log \left(\frac{n+1}{n_{0}+1}\right) \leq \log \left(\frac{2 n_{0}+\log ^{2} n_{0}+1}{n_{0}+1}\right)<\log (2.1)
$$

and

$$
\sum_{i=n_{0}+1}^{n} \frac{o(i) z_{n-i}}{\lambda^{i} z_{n}}<\log (2.2)
$$

Then $s_{n}>z_{n}(1-\log (2.2))$.

The result follows since we may choose $n_{0}$ so that all scaled coefficients $z_{n}$ are uniformly bounded away from zero for $n>n_{0}$.

We are now ready to give bounds on $s_{n} / s_{n+1}-1$ for $n$ in this initial range. Recall that this quantity will represent $D$ when we apply the Difference Argument.

Lemma 4.2. There exist constants $0<k_{1}<k_{2}$ such that if $n_{0}$ is sufficiently large, then

$$
1+\frac{k_{1}}{n_{0}}<\frac{s_{n}}{s_{n+1}}<1+\frac{k_{2}}{n_{0}} \quad \text { for } \quad n \in\left[n_{0}+\log ^{2} n_{0}, 2 n_{0}+\log ^{2} n_{0}\right] .
$$

Proof. We will rewrite the ratio $s_{n} / s_{n+1}$ as $1+\left(s_{n}-s_{n+1}\right) / s_{n+1}$ and examine the difference $s_{n}-s_{n+1}$.

First, assume $n \in\left[n_{0}+\log ^{2} n_{0}, 2 n_{0}\right]$. Then

$$
s_{n}-s_{n+1}=z_{n}-z_{n+1}-\sum_{i=n_{0}+1}^{n} \frac{o(i)}{\lambda^{i}} z_{n-i}+\sum_{i=n_{0}+1}^{n+1} \frac{o(i)}{\lambda^{i}} z_{n+1-i} .
$$

Let $n_{1}=\left\lfloor\log ^{2} n_{0}\right\rfloor$ and rewrite the two summations above as the sum of the following three terms:

$$
-\sum_{i=n_{0}+1}^{n-n_{1}} \frac{o(i)}{\lambda^{i}}\left(z_{n-i}-z_{n+1-i}\right)-\sum_{i=n-n_{1}+1}^{n}\left(\frac{o(i)}{\lambda^{i}}-\frac{o(i+1)}{\lambda^{i+1}}\right) z_{n-i}+\frac{o\left(n-n_{1}\right)}{\lambda^{n-n_{1}}} z_{n_{1}} .
$$

By estimating each term, we will show that the last term dominates.

In the first summation, all terms of the form $o(i) \lambda^{-i}$ are less than a universal constant over $n_{0}$. The differences between consecutive scaled coefficients $\left|z_{k}-z_{k+1}\right|$ form a summable series in $k$. Since the indices which occur in $\left|z_{n-i}-z_{n+1-i}\right|$ are greater than $n_{1}$, by selecting $n_{0}$ sufficiently large, we can make the sum as small as we like compared to $1 / n_{0}$.

In the second summation, $z_{n-i}$ is bounded above by a constant and the difference $\left|o(i) \lambda^{-i}-o(i+1) \lambda^{i+1}\right|$ is less than a constant over $n_{0}^{2}$. Since there are at most $\log ^{2} n_{0}$ terms in the sum, the sum is as small as we like compared to $1 / n_{0}$.

In the last term, for $n_{0}$ sufficiently large, $z_{n_{1}}$ is close to a positive constant, and $o\left(n-n_{1}\right) \lambda^{n_{1}-n}$ is approximately $1 /\left(n-\log ^{2} n_{0}\right)$. We may select constants $0<c_{1}<c_{2}$ such that for $n \in\left[n_{0}+\log ^{2} n_{0}, 2 n_{0}\right]$,

$$
\frac{c_{1}}{n_{0}}<\frac{o\left(n-n_{1}\right)}{\lambda^{n-n_{1}}} z_{n_{1}}<\frac{c_{2}}{n_{0}} .
$$


All other terms in $s_{n}-s_{n+1}$ have upper bounds which are small compared to $1 / n_{0}$. Therefore if $n_{0}$ is sufficiently large,

$$
\frac{c_{1}}{2 n_{0}}<s_{n}-s_{n+1}<\frac{2 c_{2}}{n_{0}}
$$

Finally, by Lemma 4.1 we may assume that $s_{n+1}$ is bounded away from zero. Thus we may choose constants $0<k_{1}<k_{2}$ such that

$$
1+\frac{k_{1}}{n_{0}}<1+\frac{s_{n}-s_{n+1}}{s_{n+1}}<1+\frac{k_{2}}{n_{0}} .
$$

This completes the proof in the case $n \in\left[n_{0}+\log ^{2} n_{0}, 2 n_{0}\right]$.

When $n \in\left(2 n_{0}, 2 n_{0}+\log ^{2} n_{0}\right]$, we have

$$
s_{n}=z_{n}-\sum_{i=n_{0}+1}^{n} \frac{o(i)}{\lambda^{i}} z_{n-i}+\sum_{\substack{i, j>n_{0} \\
i+j \leq n}} \frac{o(i) o(j)}{\lambda^{i+j}} z_{n-i-j}+\sum_{i=n_{0}+1}^{n / 2} \lambda^{-2 i}\left(\begin{array}{c}
o(i) \\
2
\end{array}\right) z_{n-2 i} .
$$

In the summation $\sum \lambda^{-(i+j)} o(i) o(j) z_{n-i-j}$ there are at most $\log ^{4} n_{0}$ terms. In $\sum \lambda^{-2 i}\left(\begin{array}{c}o(i) \\ 2\end{array}\right) z_{n-2 i}$, there are at most $\log ^{2} n_{0}$ terms. In both, each summand is at most a constant over $n_{0}^{2}$. For sufficiently large $n_{0}$, these terms are small compared to our dominating term. By adjusting our constants a bit, the result still follows.

4.2. An approximation of $S(t)$. To get further results for ratios $s_{n} / s_{n+1}$, we work with a power series $E(t)$ which approximates $S(t)$ and has coefficients which satisfy a useful recursion relation.

First notice

$$
\begin{aligned}
\log S(t) & =\log \left(\prod_{i=1}^{n_{0}}\left(1-t^{i}\right)^{-o(i)}\right) \\
& =-\sum_{i=1}^{n_{0}} o(i) \log \left(1-t^{i}\right) \\
& =\sum_{i=1}^{n_{0}} \frac{\operatorname{tr}\left(\Lambda^{i}\right)}{i} t^{i}+\sum_{n=n_{0}+1}^{\infty} \sum_{\substack{i \mid n \\
i \leq n_{0}}} \frac{i o(i)}{n} t^{n} .
\end{aligned}
$$

We will define $E(t)$ as the exponential of the first of the two summations above. As we will show, the exponential of the second summation has relatively small coefficients.

Letting $\tau_{n}=\lambda^{-n} \operatorname{tr}\left(\Lambda^{n}\right)$,

$$
E(t)=\sum_{n=0}^{\infty} e_{n}(\lambda t)^{n}=\exp \left(\sum_{i=1}^{n_{0}} \frac{\tau_{i}}{i}(\lambda t)^{i}\right)
$$

The recursion formula below follows from $\frac{d}{d t} E(t)=E(t) \sum_{i=1}^{n_{0}} \tau_{i} \lambda^{i} t^{i-1}$ :

$$
n e_{n}=\sum_{i=1}^{n_{0}} \tau_{i} e_{n-i} \quad \text { for } n>n_{0} .
$$

Throughout the rest of the paper, we will use the fact that $\left|\tau_{i}-1\right|$ is less than an exponentially small function of $i$.

In the following lemma, we show that $e_{n}$ approximates $s_{n}$ in an initial range. We will then use the recursion formula to produce upper and lower bounds on ratios 
$e_{n} / e_{n+1}$. This allows us to show that $e_{n}$ approximates $s_{n}$ for values of $n$ up to some exponential function of $n_{0}$.

Lemma 4.3. There exists a constant $K_{2}>0$ such that if $n_{0}$ is sufficiently large, then

$$
\left|\frac{s_{n}}{e_{n}}-1\right|<K_{2} \lambda^{-n_{0} / 3} \quad \text { for } \quad n \in\left(n_{0}, 2 n_{0}+\log ^{2} n_{0}\right] .
$$

Proof. Let

$$
L(t)=\log S(t)-\log E(t)=\sum_{n=n_{0}+1}^{\infty} \sum_{\substack{i \mid n \\ i \leq n_{0}}} \frac{i o(i)}{n} t^{n} .
$$

The coefficients of $L(t)$ are all nonnegative and are zero in degrees one through $n_{0}$. For $n_{0}$ sufficiently large and $n<n_{0}$,

$$
L_{n}=\sum_{\substack{i \mid n \\ i \leq n_{0}}} \frac{i o(i)}{n}<\lambda^{n / 2} .
$$

This follows since the indices $i$ in the above summation are less than $n / 2$ so we may assume $i o(i)<2 \lambda^{n / 2}$. Thus we have at most $n / 2$ terms in the summation all less than $2 \lambda^{n / 2} / n$.

Let $E^{\prime}(t)=\sum E_{n}^{\prime} t^{n}=\exp L(t)$. By the above inequality, $E_{n}^{\prime}$ is always less than the $n$th coefficient of the series $\exp \sum_{i=1}^{\infty} \lambda^{i / 2} t^{i}$. This series has radius of convergence $\lambda^{-1 / 2}$. Therefore, there exists a constant $C>0$ such that $E_{n}^{\prime}<C \lambda^{2 n / 3}$ for all $n$.

Since $E(t)=S(t) \exp (-L(t))$, we have

$$
e_{n}=s_{n}\left(1+\sum_{i=n_{0}+1}^{n} \frac{s_{n-i}}{s_{n}} \frac{[\exp (-L(t))]_{i}}{\lambda^{i}}\right) .
$$

The absolute value of the $i$ th coefficient of $\exp (-L(t))$ is at most $E_{i}^{\prime}$ which is bounded above by $C \lambda^{2 i / 3}$. The ratios $s_{n-i} / s_{n}$ are uniformly bounded in this region by Lemma 4.1 and the fact that the scaled coefficients have a uniform upper bound (the power series $S(t)$ has radius of convergence $1>1 / \lambda$ ). Altogether this gives a constant $K_{2}>0$ such that $\left|e_{n} / s_{n}-1\right|<K_{2} \lambda^{-n_{0} / 3}$ for $n \in\left(n_{0}, 2 n_{0}+\log ^{2} n_{0}\right]$.

The next step is to obtain upper and lower bounds on ratios of consecutive coefficients in $E(t)$. The key will be to use the recursion formula to consider the ratio $e_{n} / e_{n+1}$ as $(n+1) / n$ times a weighted sum of the previous $n_{0}$ ratios. In other words, we write

$$
\begin{aligned}
\frac{e_{n}}{e_{n+1}} & =\left(\frac{n+1}{n}\right) \frac{\sum_{i=1}^{n_{0}} \tau_{i} e_{n-i}}{(n+1) e_{n+1}} \\
& =\left(\frac{n+1}{n}\right) \sum_{i=1}^{n_{0}}\left(\frac{\tau_{i} e_{n+1-i}}{(n+1) e_{n+1}}\right) \frac{e_{n-i}}{e_{n+1-i}} .
\end{aligned}
$$

For fixed $n$, if we let $w_{i}=\tau_{i} e_{n+1-i} /(n+1) e_{n+1}$, we have $\sum_{i=1}^{n_{0}} w_{i}=1$.

We will use Lemma 4.3 and results of Section 4.1 to establish bounds on ratios of the form $e_{n} / e_{n+1}$ for $n \in\left[n_{0}+\log ^{2} n_{0}, 2 n_{0}+\log ^{2} n_{0}\right]$. Then working by induction, we will use the recursion formula to establish upper and lower bounds for $e_{n} / e_{n+1}$ for $n \in\left[n_{0}+\log ^{2} n_{0}, e^{r n_{0}}\right]$ where $r>0$ is some constant. 
Lemma 4.4. There exists a constant $k_{3}>0$ such that if $n_{0}$ is sufficiently large, then

$$
\frac{e_{n}}{e_{n+1}}>1+\frac{k_{3}}{n_{0}} \quad \text { for } \quad n \in\left[n_{0}+\log ^{2} n_{0}, \infty\right)
$$

Proof. Suppose $n_{0}$ is large enough for all previous lemmas and assume $n \in\left[n_{0}+\right.$ $\left.\log ^{2} n_{0}, 2 n_{0}+\log ^{2} n_{0}\right]$. By Lemma 4.2 $s_{n} / s_{n+1}<1+k_{1} / n_{0}$. By Lemma 4.3, the difference between $e_{n} / e_{n+1}$ and $s_{n} / s_{n+1}$ is small compared to $1 / n_{0}$. Therefore, there is a constant $k_{3}>0$ such that for $n_{0}$ sufficiently large, $e_{n} / e_{n+1}>1+k_{3} / n_{0}$ for $n$ in this initial range.

Now fix $n>2 n_{0}+\log ^{2} n_{0}$ and assume $e_{n-i} / e_{n+1-i}>1+k_{3} / n_{0}$ for $1 \leq i \leq n_{0}$. Then by the recursion formula,

$$
\frac{e_{n}}{e_{n+1}}=\left(\frac{n+1}{n}\right) \sum_{i=1}^{n_{0}} w_{i} \frac{e_{n-i}}{e_{n+1-i}}>\sum_{i=1}^{n_{0}} w_{i}\left(1+\frac{k_{3}}{n_{0}}\right)=1+\frac{k_{3}}{n_{0}} .
$$

It is somewhat more complicated to obtain an upper bound on the ratios $e_{n} / e_{n+1}$ because of the factor $(n+1) / n$ in front of the weighted sum. Our upper bound will have to grow with $n$.

The following lemma is the first which contains the choice of the value of $r$. It is important to note that in this and subsequent lemmas, $r$ is always specified before $n_{0}$ and any smaller value of $r$ would also work.

Lemma 4.5. For some $r>0$, if $n_{0}$ is sufficiently large, then

$$
\frac{e_{n}}{e_{n+1}}<1+\frac{4 \log n}{n_{0}} \quad \text { for } n \in\left[n_{0}+\log ^{2} n_{0}, e^{r n_{0}}\right]
$$

Proof. Select $r \in(0,1 / 12)$.

Reasoning in a manner similar to the previous proof, we may assume that there is a constant $k_{4}>0$ such that $e_{n} / e_{n+1}<1+k_{4} / n_{0}$ for $n \in\left[n_{0}+\log ^{2} n_{0}, 2 n_{0}+\log ^{2} n_{0}\right]$. Therefore, for $n_{0}$ sufficiently large, we can insure that $e_{n} / e_{n+1}$ is no more than $1+4 \log n / n_{0}$ for $n$ in this initial range.

Now assume that $n \in\left(2 n_{0}+\log ^{2} n_{0}, e^{r n_{0}}\right)$ and for $1 \leq i \leq n_{0}, e_{n-i} / e_{n+1-i}<$ $1+4 \log (n-i) / n_{0}$. Then by the recursion formula,

$$
\begin{aligned}
\frac{e_{n}}{e_{n+1}} & =\left(\frac{n+1}{n}\right) \sum_{i=1}^{n_{0}} w_{i} \frac{e_{n-i}}{e_{n+1-i}} \\
& <\left(\frac{n+1}{n}\right) \sum_{i=1}^{n_{0}} w_{i}\left(1+\frac{4 \log (n-i)}{n_{0}}\right) \\
& <\left(\frac{n+1}{n}\right)\left(1+\frac{4}{n_{0}} \sum_{i=1}^{n_{0}} w_{i} \log (n-i)\right) .
\end{aligned}
$$

Using facts about logarithms, we may write

$$
\sum_{i=1}^{n_{0}} w_{i} \log (n-i)<\log \left(\sum_{i=1}^{n_{0}} w_{i}(n-i)\right)=\log \left(n-\sum_{i=1}^{n_{0}} w_{i} i\right) .
$$


We will later show that $\sum_{i=1}^{n_{0}} w_{i} i>n_{0} / 3$. Assuming this, the result is proven as follows. First we observe

$$
\begin{aligned}
\frac{e_{n}}{e_{n+1}} & <\left(\frac{n+1}{n}\right)\left(1+\frac{4}{n_{0}} \log \left(n-\frac{n_{0}}{3}\right)\right) \\
& =\left(1+\frac{1}{n}\right)\left(1+\frac{4 \log n}{n_{0}}+\frac{4}{n_{0}} \log \left(1-\frac{n_{0}}{3 n}\right)\right) .
\end{aligned}
$$

We want to show that the above estimate is less than $1+4 \log n / n_{0}$, which means we want to show

$$
\frac{1}{n}+\frac{4 \log n}{n n_{0}}+\left(1+\frac{1}{n}\right) \frac{4}{n_{0}} \log \left(1-\frac{n_{0}}{3 n}\right)<0 .
$$

The above inequality follows since

$$
\left(1+\frac{1}{n}\right) \frac{4}{n_{0}} \log \left(1-\frac{n_{0}}{3 n}\right)<-\frac{4}{3 n}
$$

and $n<e^{r n_{0}}<e^{n_{0} / 12}$ implies

$$
\frac{4 \log n}{n n_{0}}<\frac{1}{3 n}
$$

It remains to show $\sum w_{i} i>n_{0} / 3$. We first note that if $n_{0}$ is sufficiently large, the weights $w_{i}$ increase for $i>\log ^{2} n_{0}$. This follows since we have a lower bound on $\tau_{i+1} / \tau_{i}$ of the form $1-C e^{-c i}$ for some constants $C, c>0$ and a lower bound on $e_{n-i} / e_{n+1-i}>1+k_{3} / n_{0}$ from Lemma 4.4

Let $n_{1}=\left\lfloor\log ^{2} n_{0}\right\rfloor$ and let $W$ denote the sum of the first $n_{1}$ weights $W=$ $\sum_{i=1}^{n_{1}} w_{i}$. By choosing $n_{0}$ sufficiently large, we can make $W$ as small as we like. This follows since $W$ is the sum of $n_{1}$ terms, all of which are no more than some universal constant times the last term $w_{n_{1}}$ (recall $w_{i}=\tau_{i} e_{n-i}, e_{n-i}$ increases with $i, \tau_{i}$ is uniformly bounded above). On the other hand, $1-W$ is the sum of $n_{0}-n_{1}$ terms all of which are greater than the weight $w_{n-1}$.

To continue, we note

$$
\frac{1}{1-W} \sum_{i=n_{1}+1}^{n_{0}} w_{i} i>\frac{1}{n_{0}-n_{1}} \sum_{i=n_{1}+1}^{n_{0}} i .
$$

This follows since in the summation on the left we have a weighted sum where increasing weights are multiplied by increasing terms, whereas the summation on the right has an even amount of weight on each term (this idea is made precise in Lemma A.1.

Now we have

$$
\sum_{i=1}^{n_{0}} w_{i} i>\frac{1-W}{n_{0}-n_{1}} \sum_{i=n_{1}+1}^{n_{0}} i=\frac{1-W}{n_{0}-n_{1}}\left(\frac{n_{0}^{2}+n_{0}}{2}-\frac{n_{1}^{2}+n_{1}}{2}\right) .
$$

By selecting $n_{0}$ sufficiently large, we can force the right-hand side to be as close as we like to $n_{0} / 2$. In particular, we can force it to be at least $n_{0} / 3$, our desired lower bound.

Our estimates on $e_{n}$ now allow us to show that the $e_{n}$ 's approximate $s_{n}$ for $n \in\left(2 n_{0}+\log ^{2} n_{0}, e^{r n_{0}}\right)$. 
Lemma 4.6. There exists a constant $K_{3}>0$ such that if $n_{0}$ is sufficiently large, then

$$
\left|\frac{s_{n}}{e_{n}}-1\right|<K_{3} \lambda^{-n_{0} / 6} \quad \text { for } \quad n \in\left[n_{0}+\log ^{2} n_{0}, e^{r n_{0}}\right] .
$$

Proof. Assume $(1+4 r)<\lambda^{1 / 6}$ and $r$ is small enough for Lemma 4.5.

Recall from the proof of Lemma 4.3 that the power series $E^{\prime}(t)$ was defined by the relation $S(t)=E(t) E^{\prime}(t)$ and there is a constant $C>0$ such that $E_{i}^{\prime}<C \lambda^{2 i / 3}$ for all $i$. We have the following:

$$
s_{n}=e_{n}\left(1+\sum_{i=n_{0}+1}^{n} \frac{e_{n-i}}{e_{n}} \frac{E_{i}^{\prime}}{\lambda^{i}}\right)<e_{n}\left(1+C \sum_{i=n_{0}+1}^{n} \frac{e_{n-i}}{e_{n}} \lambda^{-i / 3}\right) .
$$

Assume that $n_{0}$ is large enough to give bounds from all previous lemmas and assume $n \in\left[n_{0}+\log ^{2} n_{0}, e^{r n_{0}}\right]$. For estimates on the terms $e_{n-i} / e_{n}$, we have two cases.

If $n_{0}+\log ^{2} n_{0} \leq n-i<n$, then by Lemma 4.5

$$
\frac{e_{n-i}}{e_{n}}<\left(1+\frac{4 \log n}{n_{0}}\right)^{i}<(1+4 r)^{i}<\lambda^{i / 6} .
$$

Now assume $n-i<n_{0}+\log ^{2} n_{0}$. Then either $n-i \leq n_{0}$ in which case $e_{n-i}=$ $s_{n-i}$ or $n-i \in\left(n_{0}, n_{0}+\log ^{2} n_{0}\right]$ in which case Lemma 4.3 tells us that $e_{n-i}$ is approximately equal to $s_{n-i}$. Lemma 4.1 implies that there is a constant $C^{\prime}>0$ such that $s_{k}<C^{\prime} s_{\left\lfloor n_{0}+\log ^{2} n_{0}\right\rfloor}$ for all $k<n_{0}+\log ^{2} n_{0}$. Therefore, $e_{n-i} / e_{n}<2 C^{\prime} \lambda^{i / 6}$ for $n-i<n_{0}+\log ^{2} n_{0}$.

Substituting our estimates in equation (4.3), we can find a constant $K_{3}>0$ such that

$$
\left|\frac{s_{n}}{e_{n}}-1\right|<K_{3} \lambda^{-n_{0} / 6}
$$

We have now established bounds on ratios $e_{n} / e_{n+1}$ and shown that we introduce a small error by replacing $s_{n} / s_{n+1}$ with $e_{n} / e_{n+1}$. We are ready to prove the main lemma of this section. Define

$$
R(t)=\sum_{n=0}^{\infty} R_{n} t^{n}=(1-p(t)) S(t) .
$$

Polynomial Range Lemma. There exists a constant $K_{4}>0$ such that if $n_{0}$ is sufficiently large, then

$$
R_{n}<-K_{4} S_{n} / n_{0} \quad \text { for } n \in\left(n_{0}, n_{0}^{20}\right) .
$$

Proof. First assume that $n \in\left(n_{0}, 2 n_{0}+2 \log ^{2} n_{0}\right]$. From the equation

$$
R(t)=\prod_{i=n_{0}+1}^{\infty}\left(1-t^{i}\right)^{o(i)}
$$

we have

$$
R_{n}=-o(n)+\sum_{\substack{i+j=n \\
i, j>n_{0}}} o(i) o(j)+\left(\begin{array}{c}
o(n / 2) \\
2
\end{array}\right)
$$


(The last two terms occur only if $n \geq 2 n_{0}+2$, the last term occurs only if $n$ is even.)

We use estimates similar to those in Lemma 4.1 to show this expression is negative. For sufficiently large $n_{0}, o(n)>\lambda^{n} / 2 n>\lambda^{n} / 6 n_{0}$. The second term $\sum o(i) o(j)$ is the sum of no more than $\log ^{2} n_{0}$ terms, each of which is less than a constant times $\lambda^{n} / n_{0}^{2}$. The final term $\left(\begin{array}{c}o(n / 2) \\ 2\end{array}\right)$ is less than $\lambda^{n} / n_{0}^{2}$.

It also follows from the above argument that for $n_{0}$ sufficiently large, $R_{n}$ is less than a negative constant times $\lambda^{n} / n_{0}$ in this range. Since the coefficients $s_{n}=$ $\lambda^{-n} S_{n}$ are uniformly bounded, we obtain the desired upper bound of a negative constant times $S_{n} / n_{0}$.

Now fix $n \in\left(2 n_{0}+2 \log ^{2} n_{0}, n_{0}^{20}\right)$. It follows from Lemma 4.6 that the absolute value of the difference between $1-\sum_{i=1}^{d} p_{i} \lambda^{-i} s_{n-i} / s_{n}$ and $1-\sum_{i=1}^{d} p_{i} \lambda^{-i} e_{n-i} / e_{n}$ is less than a constant times $\lambda^{-n_{0} / 6}$. In particular, this difference is small compared to $1 / n_{0}$ for large $n_{0}$. It remains to show the expression $1-\sum_{i=1}^{d} p_{i} \lambda^{-i} e_{n-i} / e_{n}$ is less than a negative constant over $n_{0}$.

Let $D_{i}$ denote the difference $e_{n-i} / e_{n}-e_{n+1-i} / e_{n}$ for $1 \leq i \leq n_{0}$ and notice

$$
\frac{e_{n-i}}{e_{n}}=1+\sum_{j=1}^{i} D_{j}=1+i D_{1}+\underbrace{\sum_{j=1}^{i}(i-j)\left(D_{j+1}-D_{j}\right)}_{i \text { th error term }} .
$$

Lemma 4.4 implies $D_{1}>k_{3} / n_{0}$. Therefore, following the Difference Argument, it suffices to show that the $i$ th error term is small compared to $1 / n_{0}$ for $i=1,2, \ldots, d$.

We will work with the second order difference $D_{2}-D_{1}$. An upper bound on second order differences $D_{j+1}-D_{j}$ for $j=1,2, \ldots, d$ follows similarly. Note that we may rewrite $D_{2}-D_{1}$ in the following way:

$$
\begin{aligned}
D_{2}-D_{1}= & \left(\frac{e_{n-2}}{e_{n}}-\frac{e_{n-1}}{e_{n}}\right)-\left(\frac{e_{n-1}}{e_{n}}-1\right) \\
= & \left(\frac{(n-2) e_{n-2}}{n e_{n}}-\frac{(n-1) e_{n-1}}{n e_{n}}\right)-\left(\frac{(n-1) e_{n-1}}{n e_{n}}-\frac{n e_{n}}{n e_{n}}\right) \\
& +\frac{2}{n}\left(\frac{e_{n-2}}{e_{n}}-\frac{e_{n-1}}{e_{n}}\right) .
\end{aligned}
$$

Lemma 4.5 implies the last term above has the following upper bound:

$$
\begin{aligned}
\frac{2}{n}\left(\frac{e_{n-2}}{e_{n}}-\frac{e_{n-1}}{e_{n}}\right) & =\frac{2 e_{n-1}}{n e_{n}}\left(\frac{e_{n-2}}{e_{n-1}}-1\right) \\
& <\frac{2}{n}\left(1+\frac{4 \log n}{n_{0}}\right)\left(\frac{4 \log n}{n_{0}}\right) .
\end{aligned}
$$

Since $n \in\left(n_{0}, n_{0}^{20}\right)$, for sufficiently large $n_{0}$ this upper bound is less than a constant times $\log n_{0} / n_{0}^{2}$. 
We use the recursion formula for coefficients $e_{n}$ to rewrite the remaining terms as follows:

$$
\begin{aligned}
\frac{1}{n e_{n}} \sum_{i=1}^{n_{0}} \tau_{i}\left(e_{n-2-i}\right. & \left.-e_{n-1-i}\right)-\tau_{i}\left(e_{n-1-i}-e_{n}\right) \\
& =\frac{1}{n} \sum_{i=1}^{n_{0}} \tau_{i}\left(D_{i+2}-D_{i+1}\right) \\
& =\frac{\tau_{n_{0}} D_{n_{0}+2}}{n}+\frac{1}{n} \sum_{i=2}^{n_{0}}\left(\tau_{i-1}-\tau_{i}\right) D_{i+1}-\frac{\tau_{1} D_{2}}{n} .
\end{aligned}
$$

The first term $\tau_{n_{0}} D_{n_{0}+2} / n$ is the most difficult to get an upper bound on. It is a first order difference $\left(e_{n-n_{0}-2} / e_{n-n_{0}-1}-1\right)$ times a factor $\tau_{n_{0}} e_{n-n_{0}-1} / n e_{n}$ for which we do not yet have a sufficient upper bound. To show that this factor is small, we consider the ratio $2 e_{n-n_{0}} / n e_{n}$, which is greater than $\tau_{n_{0}} e_{n-n_{0}-1} / n e_{n}$ for $n_{0}$ sufficiently large. Using the recursion formula,

$$
\frac{e_{n-n_{0}}}{n e_{n}}=\frac{e_{n-n_{0}}}{\sum_{i=1}^{n_{0}} \tau_{i} e_{n-i}}=\frac{1}{\sum_{i=1}^{n_{0}} \tau_{i} e_{n-i} / e_{n-n_{0}}} .
$$

By Lemma 4.5] we have $e_{n-i} / e_{n-n_{0}}>\left(1+4 \log n / n_{0}\right)^{i-n_{0}}$. Therefore,

$$
\begin{aligned}
\sum_{i=1}^{n_{0}} \tau_{i} e_{n-i} / e_{n-n_{0}} & >\sum_{i=n_{0} / 2}^{n_{0}} \tau_{i}\left(1+4 \log n / n_{0}\right)^{i-n_{0}} \\
& >\frac{1}{2} \sum_{i=n_{0} / 2}^{n_{0}}\left(1+4 \log n / n_{0}\right)^{i-n_{0}} \\
& \geq \frac{1-\left(1+4 \log n / n_{0}\right)^{-n_{0} / 2}}{8 \log n / n_{0}}
\end{aligned}
$$

For $n_{0}$ sufficiently large and for $n>n_{0}$, the numerator of this last expression is at least $1 / 2$ so the entire expression is at least $n_{0} / 16 \log n$. Combining all of the bounds, we have

$$
\frac{\tau_{n_{0}} D_{n_{0}+2}}{n}<\frac{32 \log n}{n_{0}}\left(\frac{e_{n-n_{0}-2}}{e_{n-n_{0}-1}}-1\right)<\frac{128 \log ^{2} n}{n_{0}^{2}} .
$$

For $n \in\left(n_{0}, n_{0}^{20}\right)$, this is less than a constant times $\log ^{2} n_{0} / n_{0}^{2}$.

For the second term, we have $(1 / n) \sum_{i=2}^{n_{0}}\left(\tau_{i+1}-\tau_{i}\right) D_{i+1}$. Recall the differences $\left|\tau_{i+1}-\tau_{i}\right|$ are less than a $C e^{-c i}$ for some constants $C, c>0$. For $D_{i+1}$, we have an upper bound of the form

$$
D_{i+1}=\frac{e_{n-i}}{e_{n}}\left(\frac{e_{n+1-i}}{e_{n-i}}-1\right)<\left(1+\frac{4 \log n}{n_{0}}\right)^{i}\left(\frac{4 \log n}{n_{0}}\right) .
$$

Since $n<n_{0}^{20}$, by selecting $n_{0}$ sufficiently large, we can guarantee $1+4 \log n / n_{0}<$ $e^{-c / 2}$. This implies $(1 / n) \sum_{i=2}^{n_{0}}\left(\tau_{i+1}-\tau_{i}\right) D_{i+1}$ has an upper bound of the form constant times $\log n / n n_{0}<20 \log n_{0} / n_{0}^{2}$.

For the third term, we have

$$
\frac{\tau_{1} D_{2}}{n}<\tau_{1}\left(1+\frac{4 \log n}{n_{0}}\right) \frac{4 \log n}{n n_{0}}
$$


Again, for $n \in\left(n_{0}, n_{0}^{20}\right)$, this term has an upper bound of the form constant times $\log n_{0} / n_{0}^{2}$.

Therefore, $D_{2}-D_{1}$ can be written as the sum of terms, each of which is small compared to $1 / n_{0}$ for large $n_{0}$. Therefore the result follows by the Difference Argument.

\section{Polynomial to exponential Range}

In this section, we follow the Ratio Argument to show that for some $r>0$, if $n_{0}$ is sufficiently large and $n \in\left[n_{0}^{20}, e^{r n_{0}}\right)$, then the $n$th coefficient of the power series $S(t)(1-p(t))$ is bounded above by $S_{n}$ times a negative constant over $n_{0}$ (Polynomial to Exponential Range Lemma). Specifically, by Lemma 4.6 we have $s_{n-i} / s_{n} \approx$ $e_{n-i} / e_{n}$. In this section we will show that $e_{n-i} / e_{n} \approx \alpha_{n}^{-i}$ where $1-p\left(1 / \alpha_{n} \lambda\right)<0$. Then the $n$th coefficient of $S(t)(1-p(t))$ is approximately $S_{n}$ times

$$
1-\sum_{i=1}^{d} \frac{p_{i}}{\lambda^{-i}} \frac{e_{n-i}}{e_{n}} \approx 1-\sum_{i=1}^{d} p_{i}\left(\alpha_{n} \lambda\right)^{-i}=1-p\left(1 / \alpha_{n} \lambda\right)<0 .
$$

In order to prove that ratios $e_{n-i} / e_{n}$ approximate powers of $\alpha_{n}$, we make further use of the recursion formula for $e_{n}$. However, in this section we think of this relation in the following matrix form:

$$
\left(\begin{array}{c}
e_{n} \\
e_{n-1} \\
e_{n-2} \\
\vdots \\
e_{n-n_{0}+1}
\end{array}\right)=\left(\begin{array}{ccccc}
\tau_{1} / n & \tau_{2} / n & \tau_{3} / n & \cdots & \tau_{n_{0}} / n \\
1 & 0 & 0 & \cdots & 0 \\
0 & 1 & 0 & & 0 \\
\vdots & \ddots & \ddots & \ddots & \vdots \\
0 & \cdots & 0 & 1 & 0
\end{array}\right)\left(\begin{array}{c}
e_{n-1} \\
e_{n-2} \\
e_{n-3} \\
\vdots \\
e_{n-n_{0}}
\end{array}\right)
$$

Letting $A_{n}$ be the above matrix, we will get estimates on ratios $e_{n-1} / e_{n}$ for $n \geq n_{0}^{20}$ by considering ratios of entries of $A_{n} A_{n-1} \cdots A_{n-N+1} \mathbf{v}$ where $\mathbf{v}$ is an arbitrary vector with positive entries. Since

$$
\chi_{n}(t)=\operatorname{det}\left(\mathrm{I} t-A_{n}\right)=t^{n_{0}}-\tau_{1} t^{n_{0}-1} / n-\cdots-\tau_{n_{0}} / n,
$$

there is a unique positive real root $\alpha_{n}$ of $\chi_{n}(t)$ and the corresponding eigenvector of $A_{n}$ is $\left(\alpha_{n}^{n_{0}-1}, \alpha_{n}^{n_{0}-2}, \ldots, 1\right)^{\mathrm{T}}$. Our goal is to show that $e_{n-1} / e_{n}$ is approximately equal to this eigenvalue $\alpha_{n}$.

We begin by obtaining bounds on $\alpha_{n}$ and upper bounds on the other eigenvalues of $A_{n}$. Using these, we will see that for $N=n_{0}^{6}$, the vector $\left(A_{n}\right)^{N} \mathbf{v}$ is very close to a vector in the the eigenspace for $\alpha_{n}$, so ratios of consecutive entries approximate $\alpha_{n}$. Then we will need to see that applying the sequence of matrices $A_{n} A_{n-1} \cdots A_{n-N+1}$ to a vector is approximately the same as applying the $N$ th power of the single matrix $A_{n}$.

Lemma 5.1. If $n_{0}$ is sufficiently large and $n>2 n_{0}$, then

$$
\left(\frac{1}{2 n}\right)^{1 / n_{0}}<\alpha_{n}<\left(\frac{2 n_{0}}{n}\right)^{1 / n_{0}}
$$

Proof. For the lower bound on $\alpha_{n}$, we only need to select $n_{0}$ large enough so that $\tau_{n_{0}}>1 / 2$. Then

$$
\alpha_{n}^{n_{0}}=\frac{1}{n} \sum_{i=1}^{n_{0}} \tau_{i} \alpha_{n}^{n_{0}-i}>\frac{\tau_{n_{0}}}{n}>\frac{1}{2 n}
$$


For the upper bound on $\alpha_{n}$, we need $n_{0}$ to be large enough so that $\sum_{i=1}^{n_{0}} \tau_{i} / 2 n_{0}<$ 1. Then since $\sum_{i=1}^{n_{0}} \tau_{i} \alpha_{n}^{-i} / n=1$ and $\sum_{i=1}^{n_{0}} \tau_{i} / n<1$, we know $\alpha_{n}<1$. But since $\alpha_{n}<1$

$$
\alpha_{n}^{n_{0}}=\frac{1}{n} \sum_{i=1}^{n_{0}} \tau_{i} \alpha_{n}^{n_{0}-i}<\frac{1}{n} \sum_{i=1}^{n_{0}} \tau_{i}<\frac{2 n_{0}}{n} .
$$

Next, we would like an upper bound on the modulus of the other roots of the characteristic polynomial for $A_{n}$. We will prove that if $\beta_{n}$ is the root of $\chi_{n}(t)$ of the second largest modulus, then $\left|\beta_{n}\right| / \alpha_{n}<1-1 / n_{0}^{4}$. To begin we give a lower bound on the distance between the Perron root and any other root of a polynomial which has the same sign pattern as $\chi_{n}(t)$.

Lemma 5.2. Let $f(t)=t^{d}-\sum_{i=1}^{d} f_{i} t^{d-i}$ be a polynomial with $d>2$ and $f_{i}>0$ for all $i$. Let $\alpha>0$ be the unique positive real root of $f(t)$. If $\beta \neq \alpha$ is a complex number such that $f(\beta)=0$, then

$$
|\alpha-\beta|>\alpha / d \text {. }
$$

Proof. Let $g(t)=\sum_{i=1}^{d} g_{i} t^{d-i}$ be the polynomial $g(t)=f(t) /(t-\alpha)$. Equating coefficients from $(t-\alpha) g(t)$ and $f(t)$, we see $g_{d} \alpha=f_{d}>0$ and $\alpha g_{i}=f_{i}+g_{i+1}>0$ for $1 \leq i<d$.

Now let $\beta \neq \alpha$ be a complex number with $f(\beta)=0$. Then

$$
\left|\frac{g(\alpha)-g(\beta)}{\alpha-\beta}\right| \leq \frac{g(\alpha)-g(|\beta|)}{\alpha-|\beta|} .
$$

The above follows since all of the coefficients of $g(t)$ are positive and $\left|\alpha^{i}-\beta^{i}\right| /$ $|\alpha-\beta| \leq\left(\alpha^{i}-|\beta|^{i}\right) /(\alpha-|\beta|)$. By the Mean Value Theorem in $\mathbb{R}$,

$$
\frac{g(\alpha)-g(|\beta|)}{\alpha-|\beta|}=g^{\prime}(x)
$$

for some $x \in(|\beta|, \alpha)$. Since $g(t)$ has all positive coefficients and $d>2$, the function $g^{\prime}(t)$ is strictly increasing and $g^{\prime}(x)<g^{\prime}(\alpha)$.

Combining equations (5.1) and (5.2), and using $g(\beta)=0, g(\alpha)=f^{\prime}(\alpha)$ and $g^{\prime}(\alpha)=f^{\prime \prime}(\alpha) / 2$, we have the following inequality:

$$
|\alpha-\beta|>\frac{2 f^{\prime}(\alpha)}{f^{\prime \prime}(\alpha)} \text {. }
$$

To prove the claim, we need a lower bound for $2 f^{\prime}(\alpha) / f^{\prime \prime}(\alpha)$. Since $f(\alpha)=0$,

$$
f^{\prime}(\alpha)=d \alpha^{d-1}-\sum_{i=1}^{d-1}(d-i) f_{i} \alpha^{d-i-1}=\sum_{i=1}^{d} i f_{i} \alpha^{d-i-1} .
$$

Similarly,

$$
\begin{aligned}
f^{\prime \prime}(\alpha) & =d(d-1) \alpha^{d-2}-\sum_{i=1}^{d}(d-i)(d-i-1) f_{i} \alpha^{d-i-2} \\
& =\sum_{i=1}^{d}\left[2 i d-i^{2}-i\right] f_{i} \alpha^{d-i-2} .
\end{aligned}
$$


Multiplying our expression for $f^{\prime}(\alpha)$ by $2 d$ and our expression for $f^{\prime \prime}(\alpha)$ by $\alpha$, we see $2 d f^{\prime}(\alpha)>\alpha f^{\prime \prime}(\alpha)$. Therefore,

$$
|\alpha-\beta|>2 f^{\prime}(\alpha) / f^{\prime \prime}(\alpha)>\alpha / d .
$$

The above lemma gives a lower bound on the distance between $\alpha_{n}$ and a second root $\beta_{n}$ of $\chi_{n}(t)$. To get an upper bound on the modulus of $\beta_{n}$, we write

$$
\left|\frac{\beta_{n}}{\alpha_{n}}\right|^{n_{0}}=\left|\frac{\sum_{i=1}^{d} \tau_{i} \beta_{n}^{d-i}}{\sum_{i=1}^{d} \tau_{i} \alpha_{n}^{d-i}}\right|
$$

and get an upper bound on the right-hand side of the form one minus a constant over $n_{0}^{2}$. This bound follows since if we assume $\left|\beta_{n}\right|$ is close to $\alpha_{n}$, then by the previous lemma the angle $\beta_{n}$ makes with the positive real axis cannot be too small. Since $\tau_{i} \approx 1$ for large $i$, this forces enough cancellation in the sum $\sum_{i=1}^{d} \tau_{i} \beta_{n}^{d-i}$ to obtain the upper bound. We give an argument in a more general setting since it will be used again later in another context.

Lemma 5.3. Let $f_{1}, f_{2}, \ldots, f_{d}$ be a finite sequence with $d>2$ an even number and $f_{i}>0$ for all $i$. Let $\alpha>0$ be a real number and $\beta$ a complex number with $|\beta|<\alpha$. Then

$$
\left|\frac{\sum_{i=1}^{d} f_{i} \beta^{d-i}}{\sum_{i=1}^{d} f_{i} \alpha^{d-i}}\right|<1-\frac{m|\alpha-\beta|^{2}}{2 \alpha(\alpha+M)^{2}}
$$

where $m=\min f_{i+1} / f_{i}$ and $M=\max f_{i+1} / f_{i}$.

Proof. First, let $x>0$ be a real number. We claim

$$
|\alpha+x|^{2}-x|\alpha-\beta|^{2} / \alpha<|\beta+x|^{2} .
$$

Let $\theta$ be the angle that $\beta$ makes with the positive real axis. Then

$$
|\beta+x|^{2}=|\beta|^{2}+x^{2}+2 x|\beta| \cos \theta .
$$

Now consider

$$
\begin{aligned}
(\alpha+x)^{2}-x|\alpha-\beta|^{2} / \alpha & =\alpha^{2}+x^{2}+2 x \alpha-x \alpha-x|\beta|^{2} / \alpha+2 x|\beta| \cos \theta \\
& >\alpha^{2}+x^{2}+2 x|\beta| \cos \theta \\
& >|\beta+x|^{2} .
\end{aligned}
$$

This proves the claim.

Now we have for any $x>0$,

$$
\left|\frac{\beta+x}{\alpha+x}\right|<\sqrt{1-\frac{x|\alpha-\beta|^{2}}{\alpha(\alpha+x)^{2}}}<1-\frac{x|\alpha-\beta|^{2}}{2 \alpha(\alpha+x)^{2}} .
$$

To finish the proof we write

$$
\begin{aligned}
\left|\sum_{i=1}^{d} f_{i} \beta^{d-i}\right| & \leq \sum_{i=1}^{d / 2}\left|f_{2 i-1} \beta^{d-2 i+1}+f_{2 i} \beta^{d-2 i}\right| \\
& <\sum_{i=1}^{d / 2} f_{2 i-1} \alpha^{d-2 i}\left|\beta+\frac{f_{2 i}}{f_{2 i-1}}\right| .
\end{aligned}
$$


We can also write $\left|\sum_{i=1}^{d} f_{i} \alpha^{d-i}\right|=\sum_{i=1}^{d / 2} f_{2 i-1} \alpha^{d-2 i}\left|\alpha+f_{2 i} / f_{2 i-1}\right|$. Using inequality (5.3) with $r=f_{2 i} / f_{2 i-1}$, our upper bound follows.

We cannot directly apply the previous lemma to $\chi_{n}(t)$ since the coefficients $\tau_{i}$ may be zero when $i$ is small. However, we will be able to use the approximation $\tau_{i} \approx 1$ and continue.

Lemma 5.4. If $n_{0}$ is sufficiently large and $\beta_{n} \neq \alpha_{n}$ is a root of $\chi_{n}(t)=t^{n_{0}}-$ $\sum_{i=1}^{n_{0}} \tau_{i} t^{n_{0}-i} / n$, then

$$
\left|\beta_{n}\right|<\left(1-1 / n_{0}^{4}\right) \alpha_{n} \quad \text { for } \quad n \in\left(n_{0}^{4}, e^{r n_{0}}\right) .
$$

Proof. Recall, there exist constants $C, c>0$ such that $\left|\tau_{n}-1\right|<C e^{-c n}$ for all $n$. Fix $r \in(0, c / 2)$ small enough for all previous lemmas. Fix $n \in\left(n_{0}^{4}, e^{r n_{0}}\right)$. Let $\alpha=\alpha_{n}$ and $\beta=\beta_{n}$.

First, we write

$$
\left|\frac{\beta}{\alpha}\right|^{n}=\left|\frac{\sum_{i=1}^{n_{0}} \tau_{i} \beta^{n_{0}-i}}{\sum_{i=1}^{n_{0}} \tau_{i} \alpha^{n_{0}-i}}\right|
$$

and examine the effect of replacing $\tau_{i}$ by one on the right-hand side.

By Lemma 5.1, $1 / \alpha<(2 n)^{1 / n_{0}}<2^{1 / n_{0}} e^{r}$. By the way we chose $r$, for sufficiently large $n_{0}$, the sum $\left|\sum_{i=1}^{n_{0}}\left(\tau_{i}-1\right) \alpha^{n_{0}-i}\right|$ is less than a universal constant times $\alpha^{n_{0}}$. Also by Lemma 5.1, $\alpha^{n_{0}}<2 n_{0} / n<2 / n_{0}^{3}$.

If $|t|<\alpha$, it follows that the absolute value of $1-\left(\sum_{i=1}^{n_{0}} \tau_{i} t^{n_{0}-i} / \sum_{i=1}^{n_{0}} t^{n_{0}-i}\right)$ is less than a constant over $n_{0}^{3}$. Therefore, we have a constant $C^{\prime}>0$ such that

$$
\left|\frac{\beta}{\alpha}\right|^{n_{0}}<\left|\frac{\sum_{i=1}^{n_{0}} \beta^{n_{0}-i}}{\sum_{i=1}^{n_{0}} \alpha^{n_{0}-i}}\right|\left(1+\frac{C^{\prime}}{n_{0}^{3}}\right) .
$$

Combining Lemmas 5.2 and 5.3 ,

$$
\left|\frac{\sum_{i=1}^{n_{0}} \beta^{n_{0}-i}}{\sum_{i=1}^{n_{0}} \alpha^{n_{0}-i}}\right|<1-\frac{\alpha}{2 n_{0}^{2}(\alpha+1)^{2}}<1-\frac{\alpha}{8 n_{0}^{2}} .
$$

By Lemma $5.1 \alpha>2^{-1 / n_{0}} e^{-r}$; we may assume that $\alpha$ is bounded below by $1 / 2$. Altogether, we have a constant $C^{\prime \prime}>0$ such that if $n_{0}$ is sufficiently large, then

$$
\left|\frac{\beta}{\alpha}\right|^{n_{0}}<\left(1-\frac{C^{\prime \prime}}{n_{0}^{2}}\right)
$$

For sufficiently large $n_{0}$, by taking the $n_{0}$ root of both sides and noting $(1-x)^{1 / n}<1-x / n$ we have

$$
\left|\frac{\beta}{\alpha}\right|<1-1 / n_{0}^{4}
$$

Now we have estimates from Lemma 5.1 1 on the Perron eigenvalue of $A_{n}$ and from Lemma 5.4 on the second largest modulus of a root. We would like to use these to show that if $\mathbf{v}$ is a vector with nonnegative entries and $N \geq n_{0}^{6}$, then $\left(A_{n}\right)^{N} \mathbf{v}$ is close to the eigenvector for $A_{n}$. It will be convenient for us to use the matrix $B_{n}$ 
where $B_{n}$ is $A_{n}$ scaled by the Perron eigenvalue $\alpha_{n}$, i.e.,

$$
B_{n}=\left(\begin{array}{ccccc}
\tau_{1} / n \alpha_{n} & \tau_{2} / n \alpha_{n}^{2} & \tau_{3} / n \alpha_{n}^{3} & \cdots & \tau_{n_{0}} / n \alpha_{n}^{n_{0}} \\
1 & 0 & 0 & \cdots & 0 \\
0 & 1 & 0 & & 0 \\
\vdots & \ddots & \ddots & \ddots & \vdots \\
0 & \cdots & 0 & 1 & 0
\end{array}\right) .
$$

For the matrix $B_{n}$ the maximal eigenvalue is 1 and the column eigenvector is $(1,1, \ldots, 1)^{\mathrm{T}}$. If the matrix $B_{n}$ is multiplied by a vector which has the scaled coefficients $e_{n-i} \alpha_{n}^{n-i}$ in the $i$ th position for $i=1,2, \ldots, n_{0}$ the result is a vector of the scaled coefficients $e_{n-j} \alpha_{n}^{n-j}$ for $j=0,1, \ldots, n_{0}-1$.

It will also be convenient for us to use the $\infty$-norm in the following lemmas, i.e., for $\mathbf{u}=\left(u_{1}, u_{2}, \ldots, u_{n_{0}}\right),\|\mathbf{u}\|=\max \left|u_{j}\right|$.

Lemma 5.5. There exist constants $K_{5}, k_{5}>0$ such that if $n_{0}$ is sufficiently large and $n \in\left(n_{0}^{4}, e^{r n_{0}}\right)$, then the following holds. Let $\mathbf{v} \in\left(\mathbb{R}_{+}\right)^{n_{0}}$. For $j=1,2, \ldots, n_{0}$,

$$
\left[\left(B_{n}\right)^{n_{0}^{6}} \mathbf{v}\right]_{j}=a+\epsilon_{j}
$$

where $a>\|\mathbf{v}\| / n_{0}^{2}$ and $\left|\epsilon_{j}\right|<\|\mathbf{v}\| K_{5} e^{-k_{5} n_{0}}$.

Proof. Assume $r>0$ is small enough for all previous lemmas. Fix $n \in\left(n_{0}^{4}, e^{r n_{0}}\right)$. Let $B=B_{n}, \alpha=\alpha_{n}$. Let $\mathbf{v} \in\left(\mathbb{R}_{+}\right)^{n_{0}}$.

Let $\mathbf{c}=(1,1, \ldots, 1)^{\mathrm{T}}$, the eigenvector for the eigenvalue one. By Lemma 5.4 all other eigenvalues have modulus less than $1-1 / n_{0}^{4}$. Let $W$ denote the span of these complementary eigenspaces. We wish to write the vector $\mathbf{v}$ as $a \mathbf{c}+\mathbf{w}$ where $\mathbf{w} \in W$. Then we will have

$$
B^{N} \mathbf{v}=a \mathbf{c}+B^{N} \mathbf{w}
$$

and our result will follow from a lower bound on $a$ and an upper bound on $\left\|B^{N} \mathbf{w}\right\|$.

To produce the lower bound for $a$, let $\mathbf{r}=\left(r_{1}, r_{2}, \ldots, r_{n_{0}}\right)$ be the row eigenvector for 1. Here $r_{j}=\sum_{i=j}^{n_{0}} \tau_{i} \alpha^{-i} / n$. The column vector $\mathbf{r}^{\mathrm{T}}$ is orthogonal to all of $W$. To see this suppose $\beta \neq 1$ and $(\mathrm{I} \beta-B)^{m} \mathbf{x}=0$. Since $\mathbf{r}(\mathrm{I} \beta-B)=(\beta-1) \mathbf{r}$,

$$
(\beta-1)^{m} \mathbf{r x}=\mathbf{r}(\mathrm{I} \beta-B)^{m} \mathbf{x}=0 .
$$

Since $\mathbf{r}^{\mathrm{T}}$ is orthogonal to $W$ we can write

$$
\mathbf{v}=\left(\frac{\mathbf{r v}}{\mathbf{r} \cdot \mathbf{r}}\right) \mathbf{r}^{T}+\mathbf{w}_{1} \quad \text { and } \quad \mathbf{c}=\left(\frac{\mathbf{r c}}{\mathbf{r} \cdot \mathbf{r}}\right) \mathbf{r}^{T}+\mathbf{w}_{2} \quad \text { where } \mathbf{w}_{1}, \mathbf{w}_{2} \in W .
$$

Eliminating $\mathbf{r}^{\mathrm{T}}$ and solving for $\mathbf{v}$,

$$
\mathbf{v}=\left(\frac{\mathbf{r v}}{\mathbf{r c}}\right) \mathbf{c}+\mathbf{w}_{1}-\left(\frac{\mathbf{r v}}{\mathbf{r c}}\right) \mathbf{w}_{2} \text {. }
$$

Our lower bound for $a=\left(\frac{\mathbf{r v}}{\mathbf{r c}}\right)$ follows from Lemma 5.1 For $n_{0}$ sufficiently large,

$$
\mathbf{r v} \geq\|\mathbf{v}\| \min r_{j} \geq\|\mathbf{v}\| \tau_{n_{0}} \alpha^{-n_{0}} / n>\|\mathbf{v}\| / 4 n_{0}
$$

and

$$
\mathbf{r c}=\sum_{i=1}^{n_{0}} i \tau_{i} \alpha^{-i} / n<n_{0} \sum_{i=1}^{n_{0}} \tau_{i} \alpha^{-i} / n=n_{0}
$$

Therefore $a>\|\mathbf{v}\| / 4 n_{0}^{2}$.

Let $\mathbf{w}=\mathbf{w}_{1}-\left(\frac{\mathbf{r v}}{\mathbf{r c}}\right) \mathbf{w}_{2}$. Since $\mathbf{r v} \leq \mathbf{r c}\|\mathbf{v}\|$, we have $a \leq\|\mathbf{v}\|$ and $\|\mathbf{w}\| \leq$ $\|\mathbf{v}\|+a\|\mathbf{c}\| \leq 2\|\mathbf{v}\|$. 
Now we would like an upper bound for $\left\|B^{n_{0}^{6}} \mathbf{w}\right\|$. Using Lemma A.2, there is an orthonormal matrix $C$ over $\mathbb{C}$ such that $C^{\mathrm{T}} B C$ is upper triangular. The diagonal entries of this matrix are the eigenvalues of $B$ and the off-diagonal entries are at most $n_{0}$ in modulus. Without loss of generality, assume the eigenvalue one is in the $(1,1)$ entry of $C^{\mathrm{T}} B C$. Then to estimate $\left\|B^{n_{0}^{6}} \mathbf{w}\right\|$, we consider the submatrix of $C^{\mathrm{T}} B C$ which results from deleting the first row and first column. By Lemma A.3 the entries of this matrix are all less than the following expression:

$$
n_{0} n_{0}\left(\begin{array}{c}
n_{0}^{6}+n_{0}-1 \\
n_{0}
\end{array}\right)\left(1-1 / n_{0}^{4}\right)^{n_{0}^{6}-n_{0}} .
$$

By Lemma $\underline{\text { A.4. }}$

$$
\left(\begin{array}{c}
n_{0}^{6}+n_{0}-1 \\
n_{0}
\end{array}\right)<n_{0}\left(1+n_{0}^{-5}\right)^{n_{0}{ }^{6}}\left(1+n_{0}^{5}\right)^{n_{0}}
$$

and there is a constant $c>0$ such that $n_{0} n_{0}\left(\begin{array}{c}n_{0}^{6}+n_{0}-1 \\ n_{0}\end{array}\right)<e^{c n_{0} \log n_{0}}$. We know $\left(1-1 / n_{0}^{4}\right)^{n_{0}^{6}}<e^{-n_{0}^{2}}$, therefore the last term $\left(1-1 / n_{0}^{4}\right)^{n_{0}^{6}-n_{0}}$ is less than $e^{-n_{0}^{2}+1 / n_{0}^{3}}$. The term $e^{-n_{0}^{2}}$ makes the entire expression less than an exponentially small function of $n_{0}$.

Therefore, we can conclude that there exist constants $K_{5}, k_{5}>0$ such that for $n_{0}$ sufficiently large,

$$
\left\|B^{n_{0}^{6}} \mathbf{w}\right\|<K_{5}\|\mathbf{v}\| e^{-k_{5} n_{0}}
$$

It remains to show that the difference between using the matrix $\left(A_{n}\right)^{n_{0}^{6}}$ and the product $A_{n} A_{n-1} \cdots A_{n-n_{0}^{6}+1}$ is negligible. This follows fairly easily since one obtains $A_{n-k}$ from $A_{n}$ by scaling some entries by a factor of $n /(n-k)$. For large $n_{0}$, since $n \geq n_{0}^{20}$ and $k<n_{0}^{6}$, this factor is very close to one.

The estimates we need are a little more delicate than this so again it will be convenient to use the scaled matrices, but we would like to scale all matrices by the same factor. Fix $n \in\left[n_{0}^{20}, e^{r n_{0}}\right)$ and let $B_{n-i}$ denote the matrix $A_{n-i}$ scaled by $\alpha_{n}$, the eigenvalue of $A_{n}$. The maximal eigenvalue of $B_{n-i}$ will then be $\alpha_{n} / \alpha_{n-i}$.

Lemma 5.6. If $n_{0}$ is sufficiently large and $n \geq n_{0}^{20}$, then for $\mathbf{v} \in(\mathbb{R})^{n_{0}}$,

$$
\left\|\left(B_{n}\right)^{n_{0}^{6}} \mathbf{v}-B_{n} B_{n-1} \cdots B_{n-n_{0}^{6}+1} \mathbf{v}\right\|<n_{0}^{-4}\|\mathbf{v}\| .
$$

Proof. Note for any $1 \leq i \leq n_{0}^{6}$, the only nonzero row of $B_{n-i}-B_{n}$ has a sum of $\sum_{i=1}^{n_{0}} i \tau_{i} \alpha_{n}^{-i} / n(n-i)=i /(n-i)$. If $n_{0}^{20}-n_{0}^{6}>n_{0}^{16}$, this sum is less than $n_{0}^{-10}$. Therefore, $\left\|B_{n} \mathbf{v}-B_{n-i} \mathbf{v}\right\| \leq n_{0}^{-10}\|\mathbf{v}\|$.

Noting also that $\left\|B_{n-i} \mathbf{v}\right\| \leq\|\mathbf{v}\|$ for all $i$, we have

$$
\left\|\left(B_{n}\right)^{n_{0}^{6}} \mathbf{v}-B_{n} B_{n-1} \cdots B_{n-n_{0}^{6}+1} \mathbf{v}\right\| \leq n_{0}^{6} n_{0}^{-10}\|\mathbf{v}\| \leq n_{0}^{-4}\|\mathbf{v}\| .
$$

Recall our definition

$$
R(t)=(1-p(t)) S(t)
$$


Polynomial to Exponential Range Lemma. For some $r>0$, there exists a constant $K_{6}>0$ such that if $n_{0}$ is sufficiently large, then

$$
R_{n}<-K_{6} S_{n} / n_{0} \quad \text { for } n \in\left[n_{0}^{20}, e^{r n_{0}}\right) .
$$

Proof. Recall $p^{\prime}(1 / \lambda)>0$. Fix $\delta>0$ such that $|x-1|<\delta$ implies $p^{\prime}(1 / x \lambda)>$ $p^{\prime}(1 / \lambda) / 2$. Fix $r>0$ small enough so that $e^{-r}>1-\delta / 2$ and so that all previous lemmas hold.

Fix $n \in\left[n_{0}^{20}, e^{r n_{0}}\right)$. We have the following formula for $R_{n}$ :

$$
R_{n}=\lambda^{n} s_{n}\left(1-\sum_{i=1}^{d} p_{i} \lambda^{-i} \frac{s_{n-i}}{s_{n}}\right) .
$$

Lemma 4.6 implies that the difference between the above expression in parentheses and that expression with $s_{n-i} / s_{n}$ replaced by $e_{n-i} / e_{n}$ is no more than a constant times $\lambda^{-n_{0} / 6}$. We will now use the lemmas of this section to show that $e_{n-i} / e_{n} \approx$ $\alpha_{n}^{-i}$, so

$$
1-\sum_{i=1}^{d} p_{i} \lambda^{-i} \frac{e_{n-i}}{e_{n}} \approx 1-\sum_{i=1}^{d} p_{i}\left(\alpha_{n} \lambda\right)^{-i}=1-p\left(1 / \alpha_{n} \lambda\right) .
$$

Lemma 5.1 provides the lower bound $(2 n)^{-1 / n_{0}}$ for the Perron eigenvalue $\alpha_{n}$ of the matrix $A_{n}$. Since $n<e^{r n_{0}}$ we have $\alpha_{n}>2^{-1 / n_{0}} e^{-r}$. By choice of $r$, for sufficiently large $n_{0}$,

$$
1-p\left(1 / \alpha_{n} \lambda\right)<-\frac{p^{\prime}(1 / \lambda)}{2 \lambda}\left|\alpha_{n}-1\right| .
$$

Lemma 5.1 also provides an upper bound $\alpha_{n}<\left(2 n_{0} / n\right)^{1 / n_{0}}$. Since $n \geq n_{0}^{20}$, for sufficiently large $n_{0}$, we have $\alpha_{n}<1-1 / n_{0}$. Therefore, $1-p\left(1 / \alpha_{n} \lambda\right)$ is less than a negative constant over $n_{0}$.

Following the Ratio Argument, it remains to show that the approximation in equation (5.5) has an error which is small compared to $1 / n_{0}$. Let $m=n-n_{0}^{6}$, and let $\alpha=\alpha_{n}$. Let $\mathbf{v}$ denote the vector $\left(e_{m-1} \alpha^{-m+1}, e_{m-2} \alpha^{-m+2}, \ldots, e_{m-n_{0}} \alpha^{-m+n_{0}}\right)^{\mathrm{T}}$. Then there exist constants $C, c>0$ such that for $1 \leq i \leq n_{0}$,

$$
e_{n-i} \alpha^{-n+i}=a+\epsilon_{i}+\delta_{i}
$$

where $a>\|\mathbf{v}\| / 4 n_{0}^{2},\left|\epsilon_{i}\right|<C\|\mathbf{v}\| e^{-c n_{0}}($ Lemma [5.5) $)$ and $\left|\delta_{i}\right|<\|\mathbf{v}\| / n_{0}^{4}$ (Lemma 5.6).

For $n_{0}$ sufficiently large, both $\left|\epsilon_{i}\right| / a$ and $\left|\delta_{i}\right| / a$ are less than a constant over $n_{0}^{2}$. Therefore the difference between $\alpha^{i} e_{n-i} / e_{n}$ and 1 is less than a constant over $n_{0}^{2}$. Since this error is smaller than $1 / n_{0}$, the result follows from the Ratio Argument.

\section{Final FaCtorization}

In order to complete the proof, we multiply $(1-p(t)) S(t)$ by a power series of the form $(1-q(t))^{-1}$ where $q_{i}>0$ for all $i$. We wish to have the $n$th coefficient of $(1-q(t))^{-1}$ closely approximate $\beta^{n}$ for some $\beta$.

Fix $r>0$ small enough to insure the Polynomial to Exponential Range Lemma holds, $1-p\left(e^{r / 2} / \lambda\right)<0$ and $\lambda e^{-r / 2}>1$. Let $\beta=\lambda e^{-r / 2}$ and let

$$
1-q(t)=1-\sum_{i=n_{0}^{2}+1}^{n_{0}^{3}}\left\lfloor\frac{\beta^{i}}{n_{0}^{3}-n_{0}^{2}}\right\rfloor t^{i} .
$$


We will show that the Perron value for $1-q(t)$ is approximately $\beta$. Then using techniques similar to those in Section 5 (Polynomial to Exponential Range), we will show that consecutive coefficients of $(1-q(t))^{-1}$ have ratios approximately equal to $\beta$. Then, letting $U(t)=(1-q(t))^{-1}$ and $V(t)=(1-p(t)) U(t)$, we will show that for $n>n_{0}^{20}$,

$$
V_{n}=U_{n}\left(1-\sum_{i=1}^{d} p_{i} U_{n-i} / U_{n}\right) \approx U_{n}(1-p(1 / \beta))<0 .
$$

It will remain then to combine all of the lemmas to show that the $n$th coefficient of $(1-p(t)) S(t) U(t)$ is negative for all $n$.

Lemma 6.1. Let $\gamma$ denote the Perron value of $1-q(t)$. There is a constant $K_{7}>0$ such that if $n_{0}$ is sufficiently large, then

$$
|\gamma-\beta|<K_{7} \beta^{-n_{0}^{2}}
$$

Proof. First notice that $1-q(1 / \beta)>0$ which implies $\gamma<\beta$. Therefore, by the Mean Value Theorem there is a $t \in(1 / \beta, 1 / \gamma)$ such that

$$
|1 / \beta-1 / \gamma|=(1-q(1 / \beta)) /\left|q^{\prime}(t)\right| \text {. }
$$

We obtain an upper bound on $1-q(1 / \beta)$ from

$$
1-q(1 / \beta)=1-\sum_{i=n_{0}^{2}+1}^{n_{0}^{3}}\left\lfloor\frac{\beta^{i}}{n_{0}^{3}-n_{0}^{2}}\right\rfloor \beta^{-i} \leq \sum_{i=n_{0}^{2}+1}^{n_{0}^{3}} \beta^{-i} .
$$

Therefore, $1-q(1 / \beta)<\beta^{-n_{0}^{2}} /(\beta-1)$.

Now since $t>1 / \beta$ and $q(t)$ has all nonnegative coefficients,

$$
\left|q^{\prime}(t)\right|>q^{\prime}(1 / \beta)=\sum_{i=n_{0}^{2}+1}^{n_{0}^{3}} i\left\lfloor\frac{\beta^{i}}{n_{0}^{3}-n_{0}^{2}}\right\rfloor \beta^{-i+1} .
$$

Taking only the last term in the sum, for $n_{0}$ sufficiently large, we see $\left|g^{\prime}(t)\right|>\beta$. The desired inequality follows.

Now in order to estimate the coefficients of $U(t)=(1-q(t))^{-1}$, we will use the recursion relation

$$
U_{n}=\sum_{i=n_{0}^{2}+1}^{n_{0}^{3}} q_{i} U_{n-i}
$$

As in Section 5 we can think of this relation as being given by a matrix. This matrix has Perron eigenvalue $\gamma \approx \beta$, so we will be able to use the same proofs we used in Section 5 to show $U_{n} / U_{n+1} \approx 1 / \beta$. Letting $V(t)=(1-p(t)) U(t)$, this will give $V_{n} \approx U_{n}(1-p(1 / \beta))$.

Lemma 6.2. There exist constants $K_{8}, K_{9}, k_{8}>0$ such that if $n_{0}$ is sufficiently large and $n>n_{0}^{20}$, then

$$
\left|\frac{U_{n+1}}{U_{n}}-\gamma\right|<K_{8} e^{-k_{8} n_{0}^{3}}
$$

and

$$
V_{n}<-K_{9} U_{n}
$$


Proof. Let $1 / \zeta$ be any other root of $1-q(t)$. By Lemma 5.2 we know $|\gamma-\zeta|>\gamma / n_{0}^{3}$. We have bounds on the ratio of consecutive coefficients

$$
\beta\left(1-\frac{n_{0}^{3}-n_{0}^{2}}{\beta^{n_{0}^{2}}}\right)<\frac{q_{i+1}}{q_{i}}<\beta\left(1-\frac{n_{0}^{3}-n_{0}^{2}}{\beta^{n_{0}^{2}}}\right)^{-1} .
$$

Therefore using Lemma 5.3, there is a constant $C>0$ such that

$$
\left|\frac{\zeta}{\gamma}\right|^{n_{0}^{3}}=\left|\frac{\sum_{i=n_{0}^{2}+1}^{n_{3}^{3}} q_{i} \zeta^{n_{0}^{3}-i}}{\sum_{i=n_{0}^{2}+1}^{n_{3}^{3}} q_{i} \gamma^{n_{0}^{3}-i}}\right|<1-C / n_{0}^{6} .
$$

Taking the $n_{0}^{3}$ root of both sides, for sufficiently large $n_{0}$,

$$
|\zeta|<\left(1-1 / n_{0}^{12}\right) \gamma
$$

Following the argument in Lemma 5.5, there exist constants $K_{8}, k_{8}>0$ such that if we multiply an arbitrary vector $\mathbf{v}$ with nonnegative entries by the recursion matrix raised to the $n_{0}^{19}$ power, the result will be a vector $\mathbf{u}$ whose entries satisfy $\left|\mathbf{u}_{j+1} / \mathbf{u}_{j}-\gamma\right|<K_{8} e^{-k_{8} n_{0}^{3}}$. Since the recursion formula applies for all $n>n_{0}^{3}$, this gives the desired estimate on ratios of coefficients $U_{n+1} / U_{n}$ for $n>n_{0}^{20}>n_{0}^{19}+n_{0}^{3}$.

Therefore by the previous lemma, when $n_{0}$ is sufficiently large, the ratio of coefficients $U_{n-i} / U_{n}$ is as close as we like to $\beta^{-i}$ for $i \leq d$. Therefore the quantity $1-\sum_{i=1}^{d} p_{i} U_{n-i} / U_{n}$ is as close as we like to $1-p(1 / \beta)$. Since we chose $\beta$ to be a fixed amount less than $\lambda$, for some $K_{9}>0,1-\sum_{i=1}^{d} p_{i} U_{n-i} / U_{n}<-K_{9}$ and $V_{n}<-K_{9} U_{n}$.

The final step is to combine all of these estimates and show that the $n$th coefficient of $(1-p(t)) S(t) U(t)$ is always negative. For this, we consider these coefficients in two ranges.

For $n<e^{r n_{0}}$, we have $R(t)=(1-p(t)) S(t)$ and the $n$th coefficient of $R(t) U(t)$ can be written as

$$
R_{n}+U_{n_{0}^{2}} R_{n-n_{0}^{2}}+U_{n_{0}^{2}+1} R_{n-n_{0}^{2}-1}+\cdots+U_{n-n_{0}-1} R_{n_{0}+1}+U_{n} .
$$

By the lemmas from Section 4 the $R_{n}$ coefficients are negative. All coefficients of $U(t)$ are nonnegative so all coefficients in the sum above are nonpositive except $U_{n}$. To show that the entire sum is nonpositive, all we need to show is that $U_{n}$ is smaller than the absolute value of just one other term in the sum.

Then for $n>e^{3 r n_{0} / 4}$ we have $V(t)=(1-p(t)) U(t)$. The $n$th coefficient of $S(t) V(t)$ can be written as

$$
S_{n}+V_{n_{0}^{2}} S_{n-n_{0}^{2}}+V_{n_{0}^{2}+1} S_{n-n_{0}^{2}-1}+\cdots+V_{n-1} S_{1}+V_{n} .
$$

We know that all but the first $n_{0}^{20}$ terms of $V(t)$ are negative and all terms of $S(t)$ are positive. We will show that the first $n_{0}^{20}$ terms are small compared to the entire sum.

In order to show that the positive terms in the above summation do not affect the overall sign, we will need upper bounds for coefficients in $U(t)$ and in large degree terms in $S(t)$.

Lemma 6.3. The coefficient of $t^{n}$ in $U(t)=(1-q(t))^{-1}$ is less than $n \beta^{n}$ for all $n$. 
Proof. We consider the $n$th coefficient of $(1-q(t))^{-1}=\sum_{i=0}^{\infty}(q(t))^{i}$. The result follows from the fact that the coefficient of $t^{n}$ from $(q(t))^{i}$ is always less than $\beta^{n}$. This holds since the number of ways to select $i$ coefficients of $q(t)$ whose indices sum to $n$ is less than or equal to $\left(n_{0}^{3}-n_{0}^{2}\right)^{i}$.

Lemma 6.4. There exists a $\delta>0$ such that if $n_{0}$ is sufficiently large and $n>$ $e^{3 r n_{0} / 4}$, then $S_{n}<(\beta-\delta)^{n}$.

Proof. Recall $S(t)=\prod_{i=1}^{n_{0}}\left(1-t^{i}\right)^{o(i)}$. For $i$ dividing $n$, we find estimates on $\left(\begin{array}{c}o(i)+n / i-1 \\ n / i\end{array}\right)$, the $n$th coefficient of the series $\left(1-t^{i}\right)^{-o(i)}$. We use the formula

$$
\left(\begin{array}{c}
o(i)+n / i-1 \\
n / i
\end{array}\right)<n\left(1+\frac{n}{i o(i)}\right)^{o(i)}\left(1+\frac{i o(i)}{n}\right)^{n / i}
$$

from Lemma A.4 We would like to show that the logarithm of the right-hand side is less than $n \log (\beta-2 \delta)$ for $n>e^{3 r n_{0} / 4}$.

The logarithm of the right-hand side is the sum of three terms. The first, $\log n$, is clearly small compared to $n$.

For the second term, $o(i) \log (1+n / i o(i))$, there are two cases. If $i<\log n_{0}$, then

$$
o(i)<\lambda^{\log n_{0}}=n_{0}^{\log \lambda}<\left(\frac{4 \log n}{3 r}\right)^{\log \lambda} .
$$

In the case $i \geq \log n_{0}$, we use $\log (1+x)<x$ to show

$$
o(i) \log \left(1+\frac{n}{i o(i)}\right)<\frac{n}{i}<\frac{n}{\log n_{0}} .
$$

In either case, by selecting $n_{0}$ large enough the ratio of this term with $n$ is as small as we like.

For the third term, we have three cases. If $i<\log n_{0}$, then

$$
\frac{n}{i} \log \left(1+\frac{i o(i)}{n}\right)<o(i)<\left(\frac{4 \log n}{3 r}\right)^{\log \lambda} .
$$

If $i \geq \log n_{0}$ and $i o(i)<n$, then

$$
\frac{n}{i} \log \left(1+\frac{i o(i)}{n}\right)<\frac{n}{i} \log 2 \leq \frac{n \log 2}{\log n_{0}}
$$

In both of these cases, for $n_{0}$ large, the term is small compared to $n$. If $i \geq \log n_{0}$ and $i o(i) \geq n$, then we may assume $i o(i)<2 \lambda^{i}$. Therefore

$$
\frac{n}{i} \log \left(1+\frac{i o(i)}{n}\right)<\frac{n}{i} \log \left(\frac{4 \lambda^{i}}{n}\right)=n\left(\log \lambda+\frac{\log 4}{i}-\frac{\log n}{i}\right) .
$$

Recalling $\log n>3 r n_{0} / 4$, this term has an upper bound of the form

$$
\frac{n}{i} \log \left(1+\frac{i o(i)}{n}\right)<n\left(\log \lambda+\frac{\log 4}{\log n_{0}}-\frac{3 r}{4}\right) .
$$

Since $\log \beta=\log \lambda-r / 2$, in this last case, we can choose a $\delta>0$ such that for sufficiently large $n_{0}$, the term is less than $n \log (\beta-3 \delta)$.

Altogether, we may assume that the $n$th coefficient of $\left(1-t^{i}\right)^{-o(i)}$ is at most $(\beta-2 \delta)^{n}$ for $n>e^{3 r n_{0} / 4}$. The $n$th coefficient in the product $\prod_{i=1}^{n_{0}}\left(1-t^{i}\right)^{-o(i)}$ is made up of at most $\left(\begin{array}{c}n+n_{0}-1 \\ n\end{array}\right)$ terms each of which is less than $(\beta-2 \delta)^{n}$. Using 
the upper bound $\left(\begin{array}{c}n+n_{0}-1 \\ n\end{array}\right)<(2 n)^{n_{0}}$, this tells us that if $n_{0}$ is sufficiently large and $n>e^{3 r n_{0} / 4}$, then

$$
S_{n}<(2 n)^{n_{0}}(\beta-2 \delta)^{n}<(\beta-\delta)^{n} .
$$

We are now ready to combine the previous lemmas to show that the desired factorization of $1-p(t)$ over the ring of formal power series exists.

Final Factorization Lemma. There is a choice of $\beta>0$ and $n_{0} \in \mathbb{N}$ such that for

$$
q(t)=\sum_{i=n_{0}^{2}+1}^{n_{0}^{3}}\left\lfloor\frac{\beta^{i}}{n_{0}^{3}-n_{0}^{2}}\right\rfloor t^{i}
$$

the nth coefficient of the product

$$
\frac{1-p(t)}{(1-q(t)) \prod_{i=1}^{n_{0}}\left(1-t^{i}\right)^{o(i)}}
$$

is nonpositive for all $n \geq 1$.

Proof. Select $r>0$ small enough for all previous lemmas, then select $n_{0}$ large enough for all previous lemmas. For $n \in\left(0, n_{0}\right]$, the $n$th coefficient of the product $1-p(t)(1-q(t)) \prod_{i=1}^{n_{0}}\left(1-t^{i}\right)^{-o(i)}$ is zero.

For $n>n_{0}$ we follow the aforementioned argument. Recall the notation $R(t)=$ $(1-p(t)) S(t)$ and $U(t)=(1-q(t))^{-1}$. By the lemmas of Sections 4 and [5, for $n \in\left(n_{0}, e^{r n_{0}}\right)$ the $n$th coefficient $R(t)$ is nonpositive. All coefficients of $U(t)$ are nonnegative. Therefore, for $n \in\left(n_{0}, e^{r n_{0}}\right)$ it suffices to show that $U_{n}$ is smaller than the absolute value of just one term of the form $R_{i} U_{n-i}$ where $i>0$.

Region I: Fix $n \in\left(n_{0}, n_{0}^{3}\right]$. Pick $\epsilon>0$ such that $\lambda-2 \epsilon>\beta$. By the Polynomial Range Lemma, there is a constant $C_{1}>0$ such that $R_{n}<-C_{1} S_{n} / n_{0}$. Also, it follows from Lemmas 4.5 and 4.6 that there is a constant $C_{2}>0$ such that $S_{n}>C_{2}(\lambda-\epsilon)^{n}$. Therefore,

$$
\left|\frac{U_{n}}{R_{n}}\right|<\frac{n n_{0} \beta^{n}}{C_{1} C_{2}(\lambda-\epsilon)^{n}} .
$$

For $n_{0}$ sufficiently large, this is less than one.

Region II: Fix $n \in\left(n_{0}^{3}, e^{r n_{0}}\right)$. Here we compare $U_{n}$ to $U_{n-n_{0}-1} R_{n_{0}+1}$. We have $\left|R_{n_{0}+1}\right|>C_{2}(\lambda-\epsilon)^{n_{0}+1} / n_{0}$ and $U_{n}<(\beta+\epsilon)^{n_{0}+1} U_{n-n_{0}-1}$ by the results of this section. Therefore,

$$
\left|\frac{U_{n}}{R_{n_{0}+1} U_{n-n_{0}-1}}\right|<\frac{n_{0}(\beta+\epsilon)^{n_{0}+1}}{C_{2}(\lambda-\epsilon)^{n_{0}+1}} .
$$

For $n_{0}$ sufficiently large, this is less than one.

Region III: Now for $n>e^{r n_{0}}$ we change the argument. Recall the notation $V(t)=(1-p(t)) U(t)$. By Lemma 6.2 the $n$th coefficient of $V(t)$ is nonpositive for $n>n_{0}^{20}$. Since all coefficients of $S(t)$ are nonnegative it suffices to show that $V_{n}$ is larger in absolute value than $\sum_{i=0}^{n_{0}^{20}} S_{i} V_{n-i}$ where $i<n$.

Fix $n \in\left[e^{r n_{0}}, \infty\right)$. Assuming $e^{r n_{0}}>e^{3 r n_{0} / 4}+n_{0}^{20}$, by Lemma 6.4 $S_{n-i}<$ $(\beta-\delta)^{n-i}$ for $0<i<n_{0}^{20}$. Also, for $i<n_{0}^{20}$, by the results of this section, 
there exist constants $C_{3}, C_{4}>0$ such that $\left|V_{n}\right|>C_{3} U_{n}>C_{4}(\beta-\delta / 2)^{n-n_{0}^{20}}\left|V_{i}\right|$. Therefore,

$$
\left|\frac{S_{n}+\sum_{i=1}^{n_{0}^{20}} S_{n-i} V_{i}}{V_{n}}\right|<\frac{n_{0}^{20}}{C_{4}}\left(\frac{\beta-\delta}{\beta-\delta / 2}\right)^{n-n_{0}^{20}}(\beta-\delta)^{n_{0}^{20}} .
$$

For $n_{0}$ sufficiently large, this is less than one.

The above shows that we may factor $1-p(t)$ in the form appearing in the Main Reduction Lemma. This proves the remaining direction of the theorem.

\section{Appendix A. Lemmas on BINOMial COEFFicients AND MATRIX THEORY}

These results are probably known to experts in the appropriate areas but we do not have convenient references.

Lemma A.1. Suppose $w_{1} \leq w_{2} \leq \cdots \leq w_{n}$ are real numbers with $\sum_{i=1}^{n} w_{i}=1$. If $r_{1} \leq r_{2} \leq \cdots \leq r_{n}$, then

$$
\sum_{i=1}^{n} w_{i} r_{i} \geq(1 / n) \sum_{i=1}^{n} r_{i}
$$

Proof. Since the weights $w_{i}$ are increasing there is an index $j$ such that $w_{i}<1 / n$ for all $1 \leq i<j$ and $w_{i} \geq 1 / n$ for all $j \leq i \leq n$.

Notice that

$$
\sum_{i=j}^{n}\left(w_{i}-1 / n\right)=\sum_{i=1}^{j-1}\left(1 / n-w_{i}\right)
$$

Therefore

$$
\sum_{i=j}^{n}\left(w_{i}-1 / n\right) r_{i} \geq \sum_{i=1}^{j-1}\left(1 / n-w_{i}\right) r_{i} .
$$

Rearranging we obtain the desired inequality.

Lemma A.2. Let $A$ be an $n \times n$ matrix over $\mathbb{R}$. Then there is a unitary, orthonormal matrix $C$ over $\mathbb{C}$ such that $C^{\mathrm{T}} A C$ is upper triangular.

Proof. Let $\mathbf{v}_{1}, \mathbf{v}_{2}, \ldots, \mathbf{v}_{n} \in \mathbb{C}^{n}$ be a set of $\mathbb{C}$-linearly independent column eigenvectors for $A$. Apply Gram-Schmidt to these vectors to create a set of orthonormal vectors $\mathbf{u}_{1}, \mathbf{u}_{2}, \ldots, \mathbf{u}_{n_{0}-1} \in \mathbb{C}^{n_{0}}$ such that $A \mathbf{u}_{k} \in \operatorname{span}\left\{\mathbf{u}_{1}, \mathbf{u}_{2}, \ldots, \mathbf{u}_{k}\right\}$ for all $k$.

Let $C$ be the $n \times n$ matrix with columns $\mathbf{u}_{j}$. Then $C^{\mathrm{T}} A C$ is a triangular matrix since $\mathbf{u}_{i}^{\mathrm{T}} A \mathbf{u}_{j}=0$ for $i>j$.

Lemma A.3. If $A$ is an $n_{0} \times n_{0}$ triangular matrix over $\mathbb{R}_{+}$with diagonal entries less than $\beta$ and off-diagonal entries less than $K>\beta$, then the entries of $A^{N}$ are all less than $K^{n_{0}}\left(\begin{array}{c}N+n_{0}+1 \\ N\end{array}\right) \beta^{N-n_{0}}$.

Proof. The entry $A^{N}(i, j)$ is the sum of all possible products of the form

$$
A\left(i, k_{1}\right) A\left(k_{1}, k_{2}\right) \cdots A\left(k_{N-1}, j\right) .
$$

Since $A$ is triangular, only products where $i \leq k_{1} \leq k_{2} \leq \cdots \leq k_{N-1} \leq j$ are nonzero. Therefore there are at most $\left(\begin{array}{c}N+n_{0}-1 \\ N\end{array}\right)$ nonzero products in this sum. We have the bounds $A\left(k_{n}, k_{n+1}\right)<K$ for $k_{n}<k_{n+1}$ and $A\left(k_{n}, k_{n+1}\right)<\beta$ for 
$k_{n}=k_{n+1}$. There are at most $n_{0}$ terms $A\left(k_{n}, k_{n+1}\right)$ in each product with $k_{n}<k_{n+1}$. Therefore each term $A^{N}(i, j)$ is less than $K^{n_{0}}\left(\begin{array}{c}N+n_{0}-1 \\ N\end{array}\right) \beta^{N-n_{0}}$.

Lemma A.4. For all $n, k \geq 1$,

$$
\left(\begin{array}{c}
n+k-1 \\
k
\end{array}\right)<n(1+k / n)^{n}(1+n / k)^{k} .
$$

Proof. We have the following estimates on $\log (n !)=\sum_{i=1}^{n} \log i$ :

$$
\int_{1}^{n} \log x d x<\log (n !)<\int_{1}^{n+1} \log x d x
$$

Thus we obtain

$$
\begin{aligned}
\log \left(\begin{array}{c}
n+k-1 \\
k
\end{array}\right)<(n+k) & \log (n+k)-k \log k \\
& -(n-1) \log (n-1)-2 .
\end{aligned}
$$

Using the inequality $(n-1) \log (n-1)+2>(n-1) \log n$ and combining terms,

$$
\log \left(\begin{array}{c}
n+k-1 \\
k
\end{array}\right)<n \log (1+k / n)+k \log (1+n / k)+\log n .
$$

This gives the inequality.

\section{Appendix B. Lind/Marcus example}

The polynomial $1-p(t)=1-4 t+6 t^{2}-6 t^{3}$ has a factorization $\prod_{i=1}^{3}\left(1-\lambda_{i} t\right)$ where $\lambda_{1} \approx 2.57474$ and $\lambda_{2}=\overline{\lambda_{3}} \approx .71263+1.35000 i$. Lind and Marcus [LM95] Chapter 11] give $\left(\lambda_{1}, \lambda_{2}, \lambda_{3}\right)$ as an example of an $n$-tuple satisfying the BoyleHandelman conditions, but one for which there was no known primitive matrix with the corresponding nonzero spectrum. We will need a polynomial matrix $A(t)$ of at least size $4 \times 4$ to realize $1-p(t)$. To see this, suppose $A(t)$ is a $3 \times 3$ matrix with entries in $t \mathbb{Z}_{+}[t]$,

$$
A(t)=\left(\begin{array}{lll}
a(t) & b(t) & c(t) \\
d(t) & e(t) & f(t) \\
g(t) & h(t) & i(t)
\end{array}\right)
$$

such that $\operatorname{det}(\mathrm{I}-A(t))=1-p(t)$. Equating first and second degree coefficients, we see $a_{1}+e_{1}+i_{1}=4$ and $a_{1} e_{1}+a_{1} i_{1}+e_{1} i_{1} \geq 6$. This is impossible since all coefficients are nonnegative integers.

Following our factorization scheme, we begin by dividing $1-p(t)$ by powers of $1-t^{i}$. A calculation shows

$$
\begin{aligned}
& \frac{1-p(t)}{1-t}=1-3 t+3 t^{2}-3 t^{3}-3 t^{4}-3 t^{5}-3 t^{6}-3 t^{7}-3 t^{8}-\cdots, \\
& \frac{1-p(t)}{(1-t)^{2}}=1-2 t+t^{2}-2 t^{3}-5 t^{4}-8 t^{5}-11 t^{6}-14 t^{7}-17 t^{8}-\cdots, \\
& \frac{1-p(t)}{(1-t)^{3}}=1-t-2 t^{3}-7 t^{4}-15 t^{5}-26 t^{6}-40 t^{7}-57 t^{8}-\cdots
\end{aligned}
$$


The last rational power series seems to be of the correct form. In fact, we notice from the Main Reduction Lemma that it suffices to have an $N>3$ such that the coefficients of the power series $(1-p(t))(1-t)^{-1}$ and $(1-p(t))(1-t)^{-2}$ are nonpositive in degrees $N$ to $2 N$ and the coefficients of $(1-p(t))(1-t)^{-3}$ are nonpositive in degrees 1 to $N$. The value $N=4$ suffices here.

Following the scheme in the Main Reduction Lemma,

$$
\begin{aligned}
& a_{0}(t)=4 t-6 t^{2}+6 t^{3} \\
& a_{1}(t)=3 t-3 t^{2}+3 t^{3}+3 t^{4}+3 t^{5}+3 t^{6}+3 t^{7}+3 t^{8} \\
& a_{2}(t)=2 t-t^{2}+2 t^{3}+5 t^{4}+8 t^{5}+11 t^{6}+14 t^{7}+17 t^{8} \\
& a_{3}(t)=t+2 t^{3}+7 t^{4}+15 t^{5}+26 t^{6}+40 t^{7}+57 t^{8}
\end{aligned}
$$

and

$$
b_{k}(t)=\left(1-a_{k}(t)\right)(1-t)-1-a_{k-1}(t)
$$

which gives

$$
b_{1}(t)=3 t^{9}, \quad b_{2}(t)=17 t^{9}, \quad b_{3}(t)=57 t^{9} .
$$

Thus we construct the matrix

$$
A(t)=\left(\begin{array}{cccc}
t+2 t^{3}+7 t^{4}+15 t^{5}+26 t^{6}+40 t^{7}+57 t^{8} & t & 0 & 0 \\
57 t^{8} & t & t & 0 \\
17 t^{7} & 0 & t & t \\
3 t^{6} & 0 & 0 & t
\end{array}\right)
$$

with $\operatorname{det}(\mathrm{I}-A(t))=1-4 t+6 t^{2}-6 t^{3}$. Notice that $A(t)^{3}$ has no nonzero entries, i.e., $A(t)$ is primitive.

We note that in this case, one can actually use the noninteger value of $N=5 / 2$ to truncate the power series. This method results in the matrix

$$
B(t)=\left(\begin{array}{cccc}
t+2 t^{3}+7 t^{4}+15 t^{5} & t & 0 & 0 \\
15 t^{5} & t & t & 0 \\
8 t^{4} & 0 & t & t \\
3 t^{3} & 0 & 0 & t
\end{array}\right)
$$

with $\operatorname{det}(\mathrm{I}-B(t))=1-4 t+6 t^{2}-6 t^{3}$. If one writes down a primitive integer matrix which corresponds to $B(t)$ in the most straightforward way, the result is a $179 \times 179$ matrix. There are certainly smaller size matrices with the same nonzero spectrum. Determining the minimal size of a nonnegative integer matrix in this case remains an interesting open problem.

\section{REFERENCES}

[BGMY80] L. Block, J. Guckenheimer, M. Misiurewicz, and L. S. Young, Periodic points and topological entropy of one-dimensional maps, Global theory of dynamical systems (Proc. Internat. Conf., Northwestern Univ., Evanston, Ill., 1979), Lecture Notes in Math., vol. 819, Springer, Berlin, 1980, pp. 18-34. MR 82j:58097

[BH91] M. Boyle and D. Handelman, The spectra of nonnegative matrices via symbolic dynamics, Ann. of Math. (2) 133 (1991), no. 2, 249-316. MR 92d:58057 
[BH93] M. Boyle and D. Handelman, Algebraic shift equivalence and primitive matrices, Trans. Amer. Math. Soc. 336 (1993), no. 1, 121-149. MR 93e:58050

[Bor95] A. Borobia, On the nonnegative eigenvalue problem, Linear Algebra Appl. 223/224 (1995), 131-140, Special issue honoring Miroslav Fiedler and Vlastimil Pták. MR 96e:15009

[Boy93] M. Boyle, Symbolic dynamics and matrices, Combinatorial and graph-theoretical problems in linear algebra (Minneapolis, MN, 1991), IMA Vol. Math. Appl., vol. 50, Springer, New York, 1993, pp. 1-38. MR 94g:58062

[BP94] A. Berman and R. J. Plemmons, Nonnegative matrices in the mathematical sciences, Classics in Applied Mathematics, vol. 9, Society for Industrial and Applied Mathematics (SIAM), Philadelphia, PA, 1994, Revised reprint of the 1979 original. MR 95e: 15013

[Cia68] P. G. Ciarlet, Some results in the theory of nonnegative matrices, Linear Algebra and Appl. 1 (1968), no. 1, 139-152. MR 36:6434

[Fie74] M. Fiedler, Eigenvalues of nonnegative symmetric matrices, Linear Algebra and Appl. 9 (1974), 119-142. MR 51:543

[Fri78] S. Friedland, On an inverse problem for nonnegative and eventually nonnegative matrices, Israel J. Math. 29 (1978), no. 1, 43-60. MR 80h:15010

[JLL96] C. R. Johnson, T. J. Laffey, and R. Loewy, The real and the symmetric nonnegative inverse eigenvalue problems are different, Proc. Amer. Math. Soc. 124 (1996), no. 12, 3647-3651. MR 97b:15009

[Joh81] C. R. Johnson, Row stochastic matrices similar to doubly stochastic matrices, Linear and Multilinear Algebra 10 (1981), no. 2, 113-130. MR 82g:15016

[Kel71] R. B. Kellogg, Matrices similar to a positive or essentially positive matrix, Linear Algebra and Appl. 4 (1971), 191-204. MR 44:5331

[KRW97] K. H. Kim, F. W. Roush, and J. B. Wagoner, Inert actions on periodic points, Electron. Res. Announc. Amer. Math. Soc. 3 (1997), 55-62 (electronic). MR 98i:54021

[Lin84] D. A. Lind, The entropies of topological Markov shifts and a related class of algebraic integers, Ergodic Theory Dynamical Systems 4 (1984), no. 2, 283-300. MR 86c:58092

[LL79] R. Loewy and D. London, A note on an inverse problem for nonnegative matrices, Linear and Multilinear Algebra 6 (1978/79), no. 1, 83-90. MR 58:722

[LM95] D. Lind and B. Marcus, An introduction to symbolic dynamics and coding, Cambridge University Press, Cambridge, 1995. MR 97a:58050

[LM98] T. J. Laffey and E. Meehan, A refinement of an inequality of Johnson, Loewy and London on nonnegative matrices and some applications, Electron. J. Linear Algebra 3 (1998), 119-128 (electronic). MR 99f:15031

[LM99] T. J. Laffey and E. Meehan, A characterization of trace zero nonnegative $5 \times 5$ matrices, Linear Algebra Appl. 302-303 (1999), 295-302. CMP 2000:07

[Min88] H. Minc, Nonnegative matrices, Wiley-Interscience Series in Discrete Mathematics and Optimization, John Wiley \& Sons, Inc., New York, 1988, A Wiley-Interscience Publication. MR 89i:15001

[MT91] B. Marcus and S. Tuncel, The weight-per-symbol polytope and scaffolds of invariants associated with Markov chains, Ergodic Theory Dynamical Systems 11 (1991), no. 1, 129-180. MR 92g:28038

[Per53] H. Perfect, Methods of constructing certain stochastic matrices, Duke Math. J. 20 (1953), 395-404. MR 15:3h

[Per92] D. Perrin, On positive matrices, Theoret. Comput. Sci. 94 (1992), no. 2, 357366, Discrete mathematics and applications to computer science (Marseille, 1989). MR 93f:15018

[Rea94] R. Reams, Topics in matrix theory, Ph.D. thesis, National University of Ireland, Dublin, 1994.

[Rea96] R. Reams, An inequality for nonnegative matrices and the inverse eigenvalue problem, Linear and Multilinear Algebra 41 (1996), no. 4, 367-375. MR 99c:15031

[Sal72] F. L. Salzmann, A note on eigenvalues of nonnegative matrices, Linear Algebra and Appl. 5 (1972), 329-338. MR 47:8575

[Sou83] G. W. Soules, Constructing symmetric nonnegative matrices, Linear and Multilinear Algebra 13 (1983), no. 3, 241-251. MR 84m:15016 
[Sul49] H. R. Suleĭmanova, Stochastic matrices with real characteristic numbers, Doklady Akad. Nauk SSSR (N.S.) 66 (1949), 343-345. MR 11:4d

[Wuw97] G. Wuwen, Eigenvalues of nonnegative matrices, Linear Algebra Appl. 266 (1997), 261-270. MR 98h:15039

Mathematics Research Group, Alabama State University, Montgomery, Alabama 36101-0271 and Korean ACAdemy of Science and TeChnology

E-mail address: kkim@gmail.alasu.edu

Department of Mathematics, C1200, University of Texas, Austin, Texas 78712

E-mail address: ormes@math.utexas.edu

Current address: Department of Mathematics, University of Connecticut, Storrs, Connecticut 06269-3009

Mathematics Research Group, Alabama State University, Montgomery, Alabama 36101-0271

E-mail address: froush@gmail.alasu.edu 